\title{
Quantum Light Storage in Solid State Atomic Ensembles.
}

\author{
Hugues de Riedmatten, Mikael Afzelius
}

\begin{abstract}
In this chapter, we will describe the storage and retrieval of quantum light (heralded single photons and entangled photons) in atomic ensembles in a solid state environment. We will consider ensembles of rare-earth ions embedded in dielectric crystals. We will describe the methods used to create quantum light spectrally compatible with the narrow atomic transitions, as well as possible protocols based on dipole rephasing that can be used to reversibly map the quantum light onto collective atomic excitations. We will review the experimental state of the art and describe in more detail quantum light storage experiments in neodymium and praseodymium doped crystals.
\end{abstract}

\section{Introduction}

Harnessing strong and coherent interactions between quantum light and matter is an important ability in quantum science. These interactions can be used to build lightmatter interfaces enabling reversible quantum state transfer between photons and atoms. One important application of these interfaces is the realization of photonic quantum memories [1, 2, 3, 4] which allow storage of quantum information carried by photons. Quantum memories are important devices in quantum information science because they can be used as synchronization devices when many different probabilistic quantum processes are linked together. They are therefore required for scalable protocols using photons, with potential applications in optical quan-

Hugues de Riedmatten

ICFO-The Institute of Photonic Sciences, Barcelona, Spain

ICREA-Institució Catalana de Recerca i Estudis Avançats, 08015 Barcelona, Spain

e-mail: hugues.deriedmatten@icfo.es

Mikael Afzelius

Group of Applied Physics, University of Geneva, Switzerland e-mail: mikael.afzelius@ unige.ch 
tum computing, generation of multiphoton states from probabilistic pair sources [5], quantum information networks [6] and long-distance quantum communication using quantum repeaters [7, 8, 9].

The realization of quantum memories for light requires efficient and reversible mapping of photons onto long lived atomic coherences. This in turn requires strong interactions between light and matter. However, in free space the interaction between a single photon and a single atom is usually weak. One way to overcome the problem is to place the atom in a high finesse cavity which strongly enhances the interaction [10] (see chapter (Kuhn)). Another way is to use a collection of atoms, where the atom light coupling is enhanced by a factor of $\sqrt{N}$ with $N$ being the number of atoms involved. Single photons are stored in atomic ensembles as collective atomic excitation, sometimes called superatoms. These superatoms have the important property that they can be efficiently converted to single photons in a well defined spatio-temporal mode thanks to a collective interference between all the involved emitters [8]. This so-called collective enhancement is at the heart of most quantum memory protocols in atomic ensembles. The quantum control of collective atomic coherences is therefore a key task in the field of quantum memories.

For applications involving transfer of quantum information over large distances, remote quantum memories must be entangled [8, 11]. This requires that the remote quantum memories must exchange quantum information using e.g. single photons, or that photons emitted by the quantum memories interfere at a central location between the two quantum memories. If optical fibers are used, this means that quantum memories must be connected to the optical fiber network, in particular to photons at telecom wavelengths in order to minimize optical losses in the fiber transmission.

Quantum memories for light were first demonstrated in atomic gases, both roomtemperature gases and ensembles of laser-cooled atoms. Several review papers can also be found on that subject [1, 9]. Some solid-state systems offer interesting perspectives as quantum memories for light, such as rare-earth doped crystals [12, 13, 14, 15, 16], nitrogen-vacancy centers in diamond [17, 18], phonons in diamond [19, 20] and quantum dots [21, 22, 3] (see also chapter McMahon). Realizing quantum memories in solid-state systems would, in general, have several advantages, such as the absence of atomic motion and the prospects for integrated devices, which may facilitate large-scale deployment of these techniques in future quantum networks. But, controlling light-matter interactions in solid-state materials also poses important challenges, such as preserving the quantum coherence in a solid-state environment.

Here we will discuss rare-earth-ion doped crystals for quantum memories. These crystals provide a large number of atoms naturally trapped in a solid-state matrix, with spectrally narrow optical and spin transitions. Due to their particular electron level structure, they also provide exceptional coherence properties, both for the optical and spin transitions, when cooled to cryogenic temperatures.

This chapter will describe experiments and techniques developed to store nonclassical light in rare-earth-ion doped crystals. In section 2, we describe in more details the relevant properties of rare-earth-ion doped crystals and the reasons why these are interesting materials for quantum light storage. In section 3 , we describe 
quantum memory protocols that have been proposed to store quantum information in doped crystals. In section 4 , we review the experimental state-of-the-art of storing non-classical states of light in crystals. In the following two sections we describe the development of two specific sources of non-classical light (Section 5) and their application to non-classical light storage (Section 6) in $\mathrm{Pr}^{3+}$ and $\mathrm{Nd}^{3+}$ ions doped crystals. Finally, in section 7, we comment on the prospects for extending quantum light storage to longer storage times in these systems.

\section{Rare-earth-ion doped crystals}

The energy structure of ions in solid-state materials is usually strongly affected by the lattice of the host crystal, resulting in broad optical transitions with very short optical coherence time. Striking exceptions to this are rare-earth ion impurities in crystals, whose $4 \mathrm{f}-4 \mathrm{f}$ transitions were found to be extremely narrow when the first high-resolution resonance spectra were obtained in the 1970s [23]. The sharp lines are due to the shielding of the $4 \mathrm{f}$ electron shell from outermost $5 \mathrm{~s}$ and $5 \mathrm{p}$ electrons, which reduces the coupling of the $4 \mathrm{f}$ electrons to the lattice. This explains the atomic-like properties of the lanthanides in a crystal. There exist several excellent books [24, 25] and reviews of optical properties of rare-earth-ion doped crystals [23, 26, 27] and their application in quantum information science [28, 29]. Here we will summarize some properties that are particularly relevant for quantum memory applications.

The effective shielding of the $4 \mathrm{f}$ electron shell results in extremely narrow homogeneous and inhomogeneous line widths of the radiative $4 \mathrm{f}-4 \mathrm{f}$ transitions in rare-earth doped crystals. At cryogenic temperatures, the inhomogeneous broadening is the dominating broadening process, analogous to the Doppler broadening of room temperature alkali gases. But in contrast to the dynamical Doppler broadening, where atoms jump between velocity classes due to velocity changing collisions, the inhomogeneous broadening of rare-earth ion doped crystals is to a large degree static. This is a result of the physical origin of the inhomogeneous broadening [30], which can be due to local crystal strain or interactions between dopants [31]. In some cases one can observe a time-dependent broadening of a spectral channel over time, which is known as spectral diffusion [32]. However, this effect is usually weak and is taken into account by a time-dependent homogeneous line width [32]. The static inhomogeneous broadening and the large number of spectral channels that can be manipulated with precise lasers have important consequences for quantum storage experiments. These features are used in the quantum memory schemes specifically developed for these materials, resulting in capabilities difficult to obtain in gas phase experiments.

The optical inhomogeneous linewidth varies strongly between crystal hosts. In some cases the degree of broadening can be related to the ionic radius mismatch between the rare-earth dopant ion and the lattice ion that it replaces. Usual inhomo- 
geneous broadenings range from a few hundreds of $\mathrm{MHz}$ to tens of $\mathrm{GHz}$, although extreme values of around $10 \mathrm{MHz}[33$ ] and $250 \mathrm{GHz}$ have been observed [34].

The optical homogeneous linewidth can be extremely narrow (ranging from $<1$ $\mathrm{kHz}$ to $1 \mathrm{MHz}$ ) if the sample is cooled to temperatures below 5-10 K [24, 25]. Above this approximative temperature range, the homogeneous linewidth usually increases rapidly, displaying a $T^{7}$ or $T^{9}$ dependence, due to coupling to phonons (spin-lattice relaxation). Below this temperature range, due to the shielding by the $5 \mathrm{~s}$ and $5 \mathrm{p}$ electrons, the homogeneous linewidth is often limited by magnetic interactions with other rare-earth ion dopants or magnetic constituents of the lattice. This has led to the general understanding that crystal materials with low nuclear spin concentration [35, 36] and low rare-earth ion dopant concentration [32] provides a way of obtaining long coherence times, both for optical and hyperfine transitions. To increase the coherence times of hyperfine levels, it was also realized that one can exploit the non-linear magnetic Zeeman effect of the hyperfine levels in order to find sweet spots where there is a zero first-order Zeeman effect (ZEFOZ) of the hyperfine transition [37]. This effectively decouples the hyperfine transition from the fluctuating magnetic environment, which can increase the hyperfine coherence time with orders of magnitude, akin to clock transitions used in alkali atoms. In addition, one can apply dynamical decoupling schemes to further increase the spin coherence time [38, 39, 40, 41]. A recent experiment demonstrated a coherence time of 6 hours in $\mathrm{Eu}^{3+}: \mathrm{Y}_{2} \mathrm{SiO}_{5}$ [42].

The parity-forbidden $4 \mathrm{f}-4 \mathrm{f}$ transitions are only weakly allowed in crystals, and arise due to admixtures of excited configurations of different parity to the $4 \mathrm{f}$ configuration. As a consequence radiative lifetimes are long, usually in the range of 100 $\mu$ s to $10 \mathrm{~ms}$. The oscillator strengths of transitions relevant to quantum information applications are in the range of $10^{-8}$ to $10^{-6}$ [43]. The weak absorption probability of individual ions, as compared to the alkali $\mathrm{D}_{1}$ and $\mathrm{D}_{2}$ lines, is compensated by the high density of ions typical for a crystal. The doping concentrations are in the range of 10 to $1000 \mathrm{pm}$, resulting in typical number densities in the range of $10^{17}$ to $10^{19}$ ions $/ \mathrm{cm}^{3}$. The absorption coefficients for typical doping levels range from $\alpha=1$ to $50 \mathrm{~cm}^{-1}$, which often make the transitions close to opaque for a $1 \mathrm{~cm}$ long crystal.

We finally comment on the electronic ground-state substructure, which is relevant for quantum information processing in general. The $\mathrm{RE}^{3+}$ ions can be divided into Kramers and non-Kramers ions, which have odd or even number of electrons, respectively. This is relevant since the two groups react differently to the interaction with the surrounding lattice ions (crystal-field interaction). In a low-symmetry $\mathrm{RE}^{3+}$ doping site, the crystal field interaction completely lifts the electronic groundstate degeneracy for non-Kramers ions. For Kramers ions the crystal-field interaction results gives rise to a series of degenerate doublets, which is due to Kramers time-reversal symmetry. As a consequence the Kramers ions have a strong magnetic dipole moment (order of the Bohr magneton), while non-Kramers ions usually have weak nuclear magnetic moments due to the quenching of the electronic magnetic moment. In some cases, however, the nuclear moment can be strongly enhanced in non-Kramers ions through interactions induced by the crystal-field Hamiltonian. In addition we also need to consider different types of hyperfine interactions. As an 
example, in $\mathrm{Eu}^{3+}$ and $\mathrm{Pr}^{3+}$ doped crystals, which are non-Kramers ions, these ions have three hyperfine states, due to quadrupole-type interactions [24, 25]. The hyperfine level separations in these ions are of the order of 10 to $100 \mathrm{MHz}$. In Kramers ions, such as $\mathrm{Er}^{3+}$ and $\mathrm{Nd}^{3+}$, a spectrally resolved Zeeman ground-state doublet can be formed by applying a rather weak field ( $<1$ Tesla) [44]. For isotopes with non-zero nuclear spin, the interaction with unquenched electron spin results in a strong hyperfine interaction, of the order of $1 \mathrm{GHz}$ for Kramers ions [45]. These different considerations are important for different aspects of quantum memory applications, since they affect the frequencies used for state preparation of the ions and possible limitations of the memory bandwidth due to interfering optical-hyperfine transitions.

\section{Quantum memory protocols}

In this section we will shortly discuss quantum memory protocols that can be used in rare-earth-ion doped crystals. We will particularly discuss the atomic frequency comb protocol, which so far is the only protocol that has been used in these systems for storage of quantum states of light. For the reader who wants an overview of different quantum memory schemes we refer to the many excellent reviews that have been published [1, 2, 28, 3, 4].

An important class of quantum memory schemes have been inspired by the photon echo process [28], which is the optical analogue of the spin echo. In the conventional photon echo process the optical pulse to be stored is absorbed by an inhomogeneous ensemble of atoms, typically rare-earth impurity ions in a crystal. The induced atomic coherence then undergo inhomogeneous dephasing, but the dephasing can be reversed by applying an optical $\pi$-pulse a time $\tau$ after the input pulse. After a time $2 \tau$ the atomic coherences are back in phase, which results in a strong collective emission known as a photon echo. Photon echo processes have been investigated for storing and processing coherent states of light since the 1980s [28]. It was therefore natural to consider if the same processes and materials could be used to store the quantum state of a single photon. It was realized, however, that the optical $\pi$-pulse used in conventional photon echoes would cause too much spontaneous emission noise, due to the high degree of atomic excitation induced by it [46]. In 2001 Moiseev and Kröll [47] made an initial proposal for a noise free photon-echo quantum memory scheme. The scheme was proposed for Doppler-broadened lines, but sparked the interest for finding a similar scheme adapted to inhomogeneously broadened solid-state ensembles. Around 2005-2006 Nilsson and Kröll [48], Kraus et al. [49] and Alexander et al. [50] proposed a quantum memory scheme where the inhomogeneous dephasing was controlled not by a strong $\pi$-pulse, but by an external electric field gradient. The basic idea is to create a narrow spectral feature using optical pumping techniques, which is then broadened using an external field. The scheme was coined controlled reversible inhomogeneous broadening (CRIB), while a later modified version of the scheme was named gradient echo memory 
(GEM) [51]. Several experimental realizations of the CRIB/GEM storage scheme in rare-earth-ion doped crystals followed [50], [52], including storage of weak coherent states at the single photon level [13], [53]. It is worth noting that the GEM experiment reported by Hedges et al. [13] demonstrated one of the highest efficiencies reached in any quantum memory, 69\%, and the highest reached in a solid-state memory. In rare-earth-ion doped crystals the CRIB/GEM experiments were implemented using external electric fields, based on the linear Stark shift. In some systems, however, the linear Stark shift is zero due to symmetry considerations [54].

In 2008 the atomic frequency comb (AFC) quantum memory scheme was proposed [12, 55]. The motivation for the scheme was the storage of trains of pulses, so-called temporal multimode storage, which turned out to be difficult using the CRIB scheme due to the scaling of the number of modes as a function of the optical depth of the storage material. In CRIB memories the number of modes scales linearly with the optical depth $d$ of the transition [11], while for AFC it is independent of optical depth, although depending on other critical parameters, as will

(a)
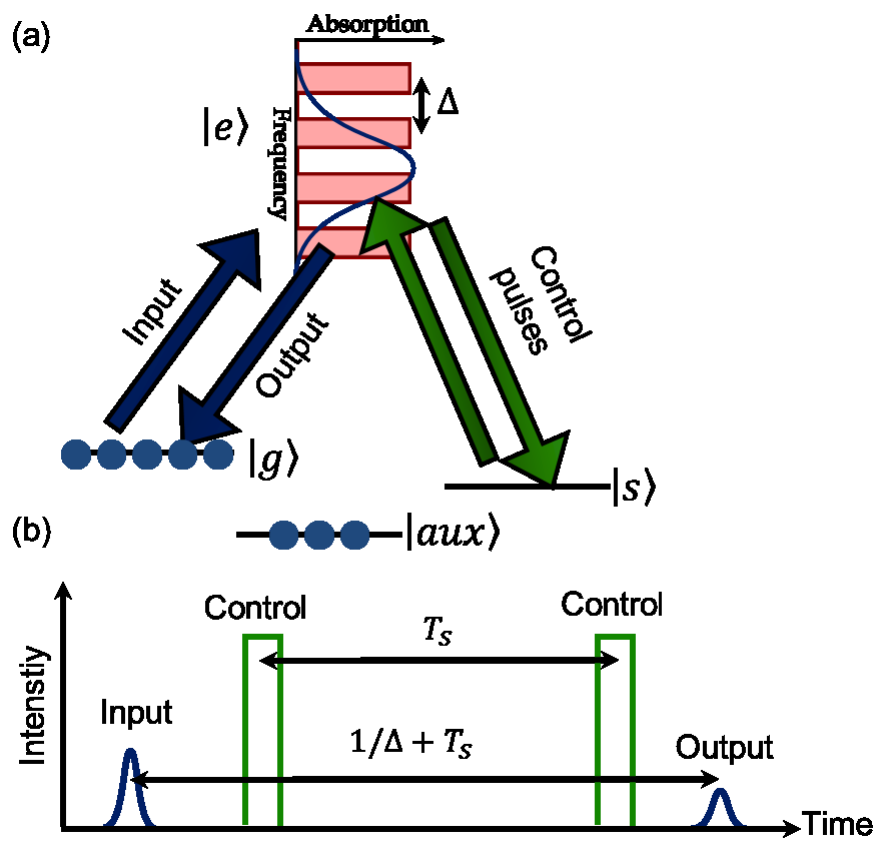

Fig. 1 (a) The input pulse is absorbed on a strongly absorbing transition whose inhomogeneous spectrum is shaped into a comb with periodicity $\Delta$. After the absorption of the input, a control pulse converts the initial optical coherence into a spin coherence, see panel (b) for the timing. Another control pulse applied a time $T_{S}$ after the first one re-establishes the optical coherence, which evolves towards an echo emission after a total storage time $1 / \Delta+T_{S}$. This scheme is the complete AFC spin-wave memory. If the control pulses are not applied, then the input will give rise to an output echo after a total storage time $1 / \Delta$, called the AFC echo scheme. We refer to the text for more details on the processes and the required energy structure. 
be discussed later. For electro-magnetically induced transparency (EIT) and Raman techniques, two common light-storage techniques, the number of modes scales as $\sqrt{d}[56]$.

The AFC scheme is based on a spectral tailoring of the inhomogeneous absorption spectrum of an optical transition $|g\rangle-|e\rangle$, where one ideally wants to make a periodic series of narrow, highly absorbing peaks. This forms the atomic frequency comb, which can be characterized by its spacing $\Delta$ and finesse $F$, in analogy with an optical cavity. A single-photon state, with a bandwidth $\gamma_{p}$ larger than $\Delta$, can be completely absorbed by the comb. Indeed, if the input pulse is short enough (meaning $\gamma_{p}>\Delta$ ), the effect of the short interaction in time is a spectral averaging of the sharp AFC structure into a smooth distribution, due to Fourier arguments, allowing for uniform absorption over the photons bandwidth. The effective optical depth of the comb is roughly $\tilde{d}=d / F[55]$, depending on the shape of the peaks, where $d$ is the peak absorption depth. The absorption probability is $1-\exp (-\tilde{d})$, showing that complete absorption can be achieved for high enough $d$ for any finesse $F$. For a more complete description, we refer the reader to [55].

Conditioned on the absorption of a single photon, the atomic state can be described by a collective Dicke state $\sum_{k} c_{k}\left|g \cdots e_{k} \cdots g\right\rangle$ [57], where the amplitudes $c_{k}$ depend on the detuning and spatial position of the particular atom $k$. These modes are initially in phase, but the collective state will rapidly dephase into a non-collective state $\sum_{k} \exp \left(-i 2 \pi \delta_{k} t\right) c_{k}\left|g \cdots e_{k} \cdots g\right\rangle$, since each term acquires an individual phase depending on the detuning $\delta_{k}$ of each excited atom. If we consider an AFC having very sharp peaks, then the detunings $\delta_{k}$ are approximately a discrete set such that $\delta_{k}=m_{k} \Delta$, where $m_{k}$ are integers. It follows that the collective state is re-established after a time $1 / \Delta$, which leads to a coherent photon-echo [58, 59, 60] type re-emission in the forward spatial mode defined by the absorbed photon. We note that the AFC echo can also be interpreted as a slow-light effect induced by the comb structure [61].

The scheme described here can only be used as a delay-line, with a fixed storage time $1 / \Delta$. In the following we will refer to this scheme as an $A F C$ echo scheme. A recent quantum repeater protocol is entirely based, however, on this scheme, but which requires heavy frequency and time multiplexing to be efficient [62]. It should also be emphasized that the AFC echo scheme provides a dynamical delay-line, which can be re-programmed with a rate related to the comb creation time. The temporal multimode capacity of the AFC scheme does not depend on optical depth, as mentioned above. The number of modes that one can store depends simply on the number of peaks in the comb, which in turn depends on the ratio of the comb periodicity $\Delta$ to the total comb bandwidth $\Gamma$.

To be able to read out a AFC memory on demand, the original proposal was based on a conversion of the optical excitation into a spin excitation [55]. This can be done by applying an optical control pulse that transfers the single optical excitation to a spin state, for instance a $\pi$-pulse, after the absorption of the single photon, but before the appearance of the AFC echo. This requires an additional ground state level $|s\rangle$, such that the states $|g\rangle,|e\rangle$ and $|s\rangle$ form a so-called $\Lambda$-system. To read out the memory a second control pulse is applied after a spin-wave storage time $T_{S}$, 
after which the collective Dicke state continues to evolve towards the AFC echo emission, after a total storage time $T_{S}+1 / \Delta$. In addition to providing on-demand read out of the memory, it can also provide a longer total storage time, since the spin coherence time can be orders of magnitude longer than the optical coherence time. In the following we will refer to this scheme as a $A F C$ spin-wave memory. The spin-wave storage requires a coherent spin-transition $|g\rangle-|s\rangle$ for storing the spin coherence, but also an additional state $|a u x\rangle$ with long population lifetime. The auxiliary ground state $|a u x\rangle$ is used for storing population that has been pumped away optically during the AFC creation process. The state $|a u x\rangle$ is also needed in the AFC echo scheme described above. The need for three ground-state levels and a coherent spin transition $|g\rangle-|s\rangle$ limits the number of known materials that can be used for spin-wave storage.

The efficiency of the complete AFC spin-wave memory depends on several factors. The most important one is the efficiency of the AFC echo, which in turn depends on the optical depth of the material, the comb parameters and the direction of recall. But one also needs to consider the efficiency of the optical control pulses and spin dephasing during the spin-wave storage time $T_{S}$. In most cases these factors act independently on the total efficiency, leading to the simple efficiency formula

$$
\eta=\eta_{A F C} \eta_{C}^{2} \eta_{S}
$$

where $\eta_{A F C}$ is the AFC echo efficiency, $\eta_{C}$ the efficiency of one optical control pulse and $\eta_{S}$ accounts for loss of efficiency due to spin decoherence. We here assume that the control pulses introduce no decoherence, we only take into account a limited transfer efficiency of population.

The AFC echo efficiency depends on the direction of recall. In forward direction the re-absorption effect in a optically dense medium limits the efficiency to $54 \%$ [63, 64]. In backward recall an interference effect makes it possible to reach $100 \%$ in principle [64, 55]. Backward recall can be achieved by using counter-propagating control pulses, but then only in spin-wave storage. The AFC echo efficiency formulas for both cases are given below

$$
\begin{gathered}
\eta_{A F C}^{f w}=\tilde{d}^{2} \exp (-\tilde{d}) \eta_{d e p h}, \\
\eta_{A F C}^{b w}=(1-\exp (-\tilde{d}))^{2} \eta_{d e p h},
\end{gathered}
$$

where $\eta_{\text {deph }}$ is a dephasing factor that accounts for the finite width and shape of the AFC teeth. It should be emphasized that these formulas also apply to the CRIB/GEM scheme. As shown in Refs [64, 55], $\eta_{\text {deph }}$ is simply the Fourier transform of a single tooth function in the comb, evaluated at the time of the AFC echo $1 / \Delta$. The effective absorption depth $\tilde{d}$ also depends on the exact shape of the AFC teeth. In Ref. [55] Gaussian peaks where considered, while in Ref. [65] formulas were given for Lorentzian shaped teeth. Later Bonarota et al. [66] showed that square peaks give the highest efficiency for a given peak optical depth $d$. For squareshaped peaks $\tilde{d}=d / F$ exactly and $\eta_{d e p h}=\operatorname{sinc}^{2}(\pi / \mathrm{F})$. 
The optical depth is in practice the most crucial parameter, which led to the proposal to put the memory in an optical cavity to enhance the effective interaction length [67],[68]. It was shown that the cavity could be operated in an optimal regime, where the input mirror reflectivity $R$ of an asymmetric cavity is tuned to the effective optical depth of the memory $\tilde{d}$ such that $R=\exp (-2 \tilde{d})$, assuming $\tilde{d}<1$, which is called an impedance-matched cavity. At the impedance-match point complete absorption can in principle be achieved, if all other losses are much smaller than $\tilde{d}$, and the efficiency is then bounded only by the intrinsic dephasing $\eta_{d e p h}$. As a consequence the cavity approach can lead to close to $100 \%$ efficiency, without resorting to the phase-matching operation required for backward recall. Note also that the impedance-matched cavity scheme can be applied to any memory scheme based on control of the inhomogeneous dephasing, e.g. AFC, CRIB or GEM. Recent experimental demonstrations of the cavity scheme reached $\mathrm{AFC}$ echo efficiencies with bright pulses of 56\% [69] in $\mathrm{Pr}^{3+}: \mathrm{Y}_{2} \mathrm{SiO}_{5}$ and $53 \%$ [70] in $\mathrm{Eu}^{3+}: \mathrm{Y}_{2} \mathrm{SiO}_{5}$, the highest reported AFC efficiencies to date.

\section{State of the art}

Although the focus of this chapter is the interaction of quantum light with rareearth doped solids, we first review a series of experiments that have been performed with weak coherent states at the single photon level. This type of experiment allows the testing of several aspects relevant for quantum light storage, in particular the coherence preservation, the noise added in the storage and retrieval processes as well as the waveform preservation. In addition, although the light at the input is classical, it has been shown that it is possible to infer the quantum character of the storage under certain conditions [13, 10, 71, 62].

The first demonstration of storage and retrieval of light at the single photon level in a solid state device, which was also the first demonstration of the AFC echo scheme, was done in 2008 at the University of Geneva [12]. Weak coherent light pulses were stored for up to $1 \mu \mathrm{s}$ using the atomic frequency comb scheme in a $\mathrm{Nd}^{3+}: \mathrm{YVO}_{4}$ crystal. Single photon level time-bin qubits were also stored and the coherence was shown to be preserved to a high degree during the storage and retrieval process. Finally, a proof of principle experiment of temporal multimodality of the protocol was done, with the storage and retrieval of 4 temporal modes. The storage and retrieval efficiency was $<1 \%$ in that initial demonstration. However, several other single photon level experiments in other materials have since then demonstrated the AFC echo scheme at the single photon level with much higher efficiencies, reaching $9 \%$ efficiency in $\mathrm{Tm}^{3+}: \mathrm{YAG}$ [65] and $25 \%$ in $\mathrm{Pr}^{3+}: \mathrm{Y}_{2} \mathrm{SiO}_{5}$ [72, 73].

Another aspect that has been improved in recent experiments is the multimode capacity. The reversible mapping of up to 64 weak pulse temporal modes has been demonstrated in a Nd:YSO crystal [74]. The coherence was verified by simultaneously storing and analyzing multiple time-bin qubits. It has also been shown that, 
combined with phase modulators, AFC can be used as a programmable processor for spectral and temporal manipulation of single qubits [75]. Hong-Ou-Mandel interference between two AFC echoes recalled from Tm doped waveguides has been demonstrated [76]. Finally, the AFC storage was also extended to polarization qubits [71, 77, 78], and more recently to spectrally multiplexed time-bin qubits with selective readout in frequency [62]. Note that for all AFC echo experiments mentioned above, the storage was done in the excited state only, leading to short and pre-determined storage time. The CRIB/GEM protocol allows on demand read-out even with storage in the excited state, as has been demonstrated at the single photon level in an Erbium doped crystal [53] and in a Pr doped crystal [13]. The latter experiment reported the highest efficiency for any solid state memory so far $(69 \%)$. It also showed that the memory operated in the quantum regime, meaning that the storage and retrieval fidelity was measured to be higher than the one achievable with a classical memory.

The first demonstration of quantum light storage in solid state device was reported simultaneously in 2011 by two groups, one from the University of Geneva [14] and one from the University of Calgary [15]. Both experiments demonstrated the storage of entangled photons in a rare-earth doped crystal, using the AFC echo scheme. Both experiments created compatible photon pair sources with one photon matching the storage device and the other photon at telecommunication wavelength. The two teams used different storage media with different properties and bandwidth. The Calgary experiment used a broadband AFC in a Tm doped Lithium Niobate waveguide absorbing light at $793 \mathrm{~nm}$. One photon of the pair was stored for $7 \mathrm{~ns}$ in the waveguide, with a storage and retrieval efficiency of $2 \%$ ( excluding coupling losses in the waveguide). It was also shown that time-bin entanglement was preserved during the storage and retrieval, and a violation of a Bell inequality was demonstrated between the telecom photon and the stored and retrieved photon. The Geneva experiment used a Nd doped crystal absorbing at $883 \mathrm{~nm}$, with a storage bandwidth of $120 \mathrm{MHz}$ and featured a maximal storage time of $200 \mathrm{~ns}$ and a maximal efficiency of $20 \%$. The preservation of energy-time entanglement was demonstrated. These experiments will be described in more detail in section 6.1

These experiments demonstrated for the first time entanglement between a telecom photon and a collective optical atomic excitation in a solid state device. It should be noted however that in both cases the photons were stored as optical atomic excitations, leading to short and only pre-determined storage times.

Following these initial experiments, further developments by the same groups included the storage and retrieval of polarization [77] and time-bin qubits [79] carried by heralded single photons. The Geneva group also reported an experiment demonstrating entanglement between two crystals [80] (it will be described in more detail in section 6.1), followed by a experiment demonstrating quantum teleportation of a telecom wavelength photon onto a collective atomic optical excitation [81]. Furthermore, the quantum storage of a 3-dimensional orbital-angular-momentum entangled photon has been reported in a $\mathrm{Nd}^{3+}: \mathrm{YVO}_{4}$ crystal by a group in Hefei [82].

In 2014 an experiment demonstrating quantum storage of heralded single photons using the AFC echo scheme in a Praseodymium doped crystal absorbing at 606 
$\mathrm{nm}$ was carried out at ICFO. This material has demonstrated promising properties in the storage of classical light, including long storage times up to 1 minute [83, 41] and high storage and retrieval efficiencies as mentioned above [13]. Contrary to materials used in previous demonstrations, it also possesses 3 ground state levels, such that spin-wave storage is in principle possible. However this comes with the drawback that the spacing between the hyperfine states is small, therefore limiting the storage bandwidth to a few $\mathrm{MHz}$. This poses strong challenges for the realization of a suitable quantum light source. The realization of such a source [84] and the storage experiment [16] will be described in more detail in sections 5.3 and 6.2 , respectively.

While the previous experiments have been performed in rare-earth doped crystals, a recent experiment demonstrated that it is also possible to store non classical light states in an amorphous environment. An erbium-doped standard telecom glass fiber was used as storage device, and photons at telecom wavelengths were stored using the atomic frequency comb scheme, with an efficiency around $1 \%$ and a storage time of $5 \mathrm{~ns}$ [85]. The experiment also showed that entanglement was preserved during the storage in the fiber.

Finally, we shortly mention experiments aiming at using crystals as a source of photon pairs with embedded memory. In these protocols, the crystal is illuminated with classical pulses, creating non classical correlations between an emitted photon and a stored collective atomic excitation. The protocols include the rephasing of amplified spontaneous emission (RASE) [86] and a combination of the DLCZ and AFC scheme [87]. First demonstrations of the RASE scheme have been realized, with strong but still classical correlations obtained in $\mathrm{Pr}^{3+}: \mathrm{Y}_{2} \mathrm{SiO}_{5}[88]$ in the photon counting regime, and evidence of non-classical correlations obtained with homodyne detection in $\mathrm{Tm}^{3+}:$ YAG [89].

\section{Quantum Light sources compatible with solid state quantum memories}

In order to achieve strong interactions between a single photon and a crystal, and to achieve high efficiency storage, it is crucial that the quantum light has spectral properties that match those of the quantum memory. The bandwidth of AFC memories is given by the width of the AFC that can be created in the crystal. For obtaining the high-finesse combs necessary to achieve high efficiency storage, the width of the comb is limited by the spacing between the adjacent states in the ground or excited state manifolds. Note that AFC broader than the spacing between ground and excited states can be created, however with a low finesse leading to limited storage efficiencies, see e.g. [15, 90]. In the case where the spacing becomes bigger than the inhomogeneous broadening of the optical transition, the limit is then given by the latter. This situation could be encountered with Kramers ions (e.g. $\mathrm{Nd}^{3+}, \mathrm{Er}^{3+}$ ), where moderate magnetic fields could split the states by several $\mathrm{GHz}$. In principle, high efficiency storage using the AFC echo scheme could therefore reach GHz band- 
width. In practice however, creating high quality combs for long storage times over such a large bandwidth is experimentally challenging. For achieving spin-wave storage, non-Kramers ions such as Pr and Eu are good candidates. In these materials, the spacing between hyperfine states are much smaller, leading to much smaller bandwidths, which can be as low as a few tens of $\mathrm{MHz}$ for $\mathrm{Eu}^{3+}: \mathrm{Y}_{2} \mathrm{SiO}_{5}$ and $4.6 \mathrm{MHz}$ for the excited state of $\operatorname{Pr}^{3+}: \mathrm{Y}_{2} \mathrm{SiO}_{5}$. The creation of single photons with such a narrow linewidth is experimentally challenging. Designing and implementing narrowband quantum light sources [91, 92, 93, 94, 95] that can be interfaced with atoms has been the subject of several investigations in recent years [96, 97, 98, 99, 100, 101]. (see also chapters (Chih-Sung Chuu, Mitchell, Zhao)).

The quantum light sources compatible with solid-state quantum memories that have been realized up to now make use of spontaneous parametric down conversion (SPDC), where a pump photon is probabilistically split into a photon pair, with energy and momentum conservation. A great advantage of this solution is that it is very flexible in terms of wavelengths of the created photons, which allows the coupling to quantum memories operating at any wavelengths. Moreover, it can create two photons with different frequencies, which can be used for example to create non classical correlations between a quantum memory operating in the visible range and a photon at telecommunication wavelengths. However, the spectrum of the photons emitted by spontaneous down conversion typically goes from $100 \mathrm{GHz}$ to $\mathrm{THz}$, several orders of magnitude larger than the bandwidth of quantum memories. Therefore extensive filtering must be applied in order to generate quantum memory compatible quantum light using spontaneous down conversion.

Filtering can be applied after the source, using passive filters. However this requires extremely bright sources [14], e.g. waveguide sources. The waveguide increases the production rate of photon pairs significantly [102], allowing for an efficient source while pumping it with a low peak-power cw laser. This is particularly important for a strongly filtered source, in order to have a sufficiently high probability of creating a photon pair within the filtered spectral regions. It is important to note that when passive filtering is used, the number of photon pairs per coherence time (or the spectral brightness expressed in pairs per second per $\mathrm{mW}$ of pump power and per $\mathrm{MHz}$ of bandwidth) does not change with the filter width [103]. While the rate of created pairs per second decreases with the filter width, the coherence time of the photon increases, leading to a constant spectral brightness.

Another way of implementing a narrow-band quantum light source from spontaneous down conversion is to insert the non linear crystal in an optical cavity [91] (see also chapters (Zhao,Mitchell)). This not only has the advantage of providing filtering, but also enhances the probability of generating a photon within a cavity mode, with respect to the no cavity case. In the ideal case, the enhancement is given by $Q=F^{3} /\left(\pi F_{0}\right)$ [91], where $F$ is the finesse of the cavity and $F_{0}$ is the finesse calculated only from the mirrors reflectivity. In order to reach this enhancement, both signal and idler fields need to be resonant with the cavity.

Beyond the use of SPDC, several other systems could be used as quantum light sources. It could be possible to use the doped crystals themselves as sources of 
photon pairs with the required spectral properties, as shown with quantum memories based on atomic gases (see. e.g. [9] for a review). However, this turns out to be much more difficult to implement in rare-earth doped solids, due to the very small oscillator strength of the optical transition. Several schemes have been proposed, including the rephasing of amplified spontaneous emission (RASE) [86] and a combination of the DLCZ and AFC scheme [87]. Solid state single photon emitters may also be used as compatible single photon sources, e.g. quantum dots or single molecules. Quantum dots have typically $\mathrm{GHz}$ spectral bandwidths (see chapters Lanco, Schneider,McMahon), which may be compatible with the AFC echo scheme with broadband combs. The challenge is to tune the quantum dot in resonance with the rare-earth ensemble, without broadening the line. Single molecules in solid state matrices have shown much narrower spectral bandwidth, down to tens of $\mathrm{MHz}$ [104], which could be used with spin-wave quantum memories. Again, the challenge is to tune them near resonance. A potential solution for the frequency mismatch is to implement quantum frequency conversion [105] which has been used recently to interface telecom photons to quantum memories [106, 73].

\subsection{Characterizing photon pair sources}

In this section, we discuss various ways to characterize photons pairs emitted by SPDC and to quantify the correlations between signal and idler fields. The state created by single mode SPDC is given by (for $p \ll 1$ ):

$$
|\Psi\rangle_{s, i}=\sqrt{1-p} \sum_{n=0}^{\infty} p^{\frac{n}{2}}|n\rangle_{s}|n\rangle_{i}
$$

where $p$ is the probability to create a photon pair and is proportional to the pump power, and $|n\rangle_{s}\left(|n\rangle_{i}\right)$ is a n photon Fock state in the signal (idler) mode. This state is known as two-mode squeezed state. It displays very strong quantum correlations between the two modes, i.e. the signal and idler fields.

For a photon pair source, the quality of the correlations between signal and idler fields is usually quantified measuring the second order cross-correlation function $G_{s, i}^{(2)}(\tau)$ between the two fields, by performing a coincidence measurement. The normalized form of $G_{s, i}^{(2)}(\tau)$, denoted as $g_{s, i}^{(2)}(\tau)$ can be expressed as:

$$
g_{s, i}^{(2)}(\tau) \equiv \frac{\left\langle E_{s}^{\dagger}(t) E_{i}^{\dagger}(t+\tau) E_{i}(t+\tau) E_{s}(t)\right\rangle}{\left\langle E_{i}^{\dagger}(t+\tau) E_{i}(t+\tau)\right\rangle\left\langle E_{S}^{\dagger}(t) E_{s}(t)\right\rangle},
$$

where $E_{s, i}^{\dagger}\left(E_{s, i}\right)$ is the electric field creation (annihilation) operator for the signal and idler fields. For the ideal two mode squeezed state of Eq. 4. the cross-correlation function is given by :

$$
g_{s, i}^{(2)}=1+\frac{1}{p}
$$


We see that the correlation decreases when increasing $p$. This is the consequence of the creation of multiple pairs. We also see that $g_{s, i}^{(2)}$ can become arbitrarily high for low excitation probability. However, this comes at the expense of the count rate, since the mean number of photons in the signal mode is given by

$$
\bar{n}_{s}=\frac{p}{1-p}
$$

This illustrates a fundamental limitation of SPDC: there is a trade off between the degree of quantum correlation and the count rate that can be obtained. In practice, $g_{s, i}^{(2)}$ is determined over a detection window $\Delta \tau$ as :

$$
g_{s, i}^{(2)}(\Delta \tau)=\frac{p_{s, i}}{p_{s} p_{i}}
$$

where $p_{s, i}$ is the probability to detect a coincidence between signal and idler photons and $p_{s}\left(p_{i}\right)$ is the probability to detect a signal (idler) photon in a time interval $\Delta \tau$.

The second order cross-correlation function can also be used to gain information on the spectral content of the photon pairs. For example, in the case of doublyresonant cavity-enhanced downconversion, following the theory used in [99, 101], $g_{s, i}^{(2)}(\tau)$ takes the form:

$$
\begin{aligned}
g_{s, i}^{(2)}(\tau) \propto & \mid \sum_{m_{s}, m_{i}=0}^{\infty} \frac{\sqrt{\gamma_{s} \gamma_{i} \omega_{s} \omega_{i}}}{\Gamma_{s}+\Gamma_{i}} \\
& \times\left\{\left.\begin{array}{ll}
e^{-2 \pi \Gamma_{s}\left(\tau-\left(\tau_{0} / 2\right)\right)} \operatorname{sinc}\left(i \pi \tau_{0} \Gamma_{s}\right) & \tau \geq \frac{\tau_{0}}{2} \\
e^{+2 \pi \Gamma_{i}\left(\tau-\left(\tau_{0} / 2\right)\right)} \operatorname{sinc}\left(i \pi \tau_{0} \Gamma_{i}\right) & \tau<\frac{\tau_{0}}{2}
\end{array}\right|^{2},\right.
\end{aligned}
$$

where $\gamma_{s, i}$ are the cavity damping rates for signal and idler, $\omega_{s, i}$ are the central frequencies, $\Gamma_{s, i}=\gamma_{s, i} / 2+i m_{s, i} \mathrm{FSR}_{\mathrm{s}, \mathrm{i}}$ with mode indices $m_{s, i}$ and free spectral ranges $\mathrm{FSR}_{\mathrm{s}, \mathrm{i}}$, and $\tau_{0}$ is the transit time difference between the signal and idler photons through the SPDC crystal. Eq. 9 shows that the second order cross-correlation function for a multimode cavity output displays an oscillatory behavior with peaks separated by the inverse of the cavity free spectral range. The width of the peaks is directly related to the number of spectral modes in the signal and idler fields. For two modes, the oscillation will be sinusoidal, and the width will then decrease with the number of modes. In practice, the minimum width that can be detected is given by the detectors' time resolution.

For the single mode case, we have :

$$
g_{s, i}^{(2)}(\tau)=1+\frac{4}{p} \frac{\gamma_{s} \gamma_{i}}{\left(\gamma_{s}+\gamma_{i}\right)^{2}} f(\tau)
$$

where 


$$
f(\tau)= \begin{cases}e^{-\gamma_{s} \tau} & \tau \geq 0 \\ e^{\gamma_{i} \tau} & \tau<0\end{cases}
$$

This is the same expression obtained for the case without cavity, but with single mode Lorentzian filters with width $\gamma_{s}$ and $\gamma_{i}$ inserted in the signal and idler modes, respectively [107].

Information about the spectral content of the created photons can also be obtained by measuring the unconditional second order autocorrelation function $g_{s, s}^{(2)}$ and $g_{i, i}^{(2)}$, for the signal and idler fields, respectively. For an ideal two mode squeezed states, the unconditional field exhibit thermal statistics with $g_{s, s}^{(2)}(0)=g_{i, i}^{(2)}(0)=2$. However, if several spectral modes are present, it has been shown [108] that the autocorrelations decrease as $g_{s, s}^{(2)}(0)=g_{i, i}^{(2)}(0)=1+1 / K$ where $K$ is the number of modes. By measuring the unconditional second order autocorrelation function, one can therefore bound the number of spectral modes present in the fields. This is valid as long as the measurement is not limited by noise.

The non-classical nature of the correlation between signal and idler fields can be experimentally assessed with a Cauchy-Schwarz inequality. For a pair of independent classical fields the following inequality must be fulfilled:

$$
R=\frac{\left(g_{s, i}^{(2)}\right)^{2}}{g_{s, s}^{(2)} g_{i, i}^{(2)}} \leq 1
$$

If the signal and idler fields exhibit thermal or sub-thermal statistics $\left(g_{s, s}^{(2)}(0)=\right.$ $g_{i, i}^{(2)}(0) \leq 2$, the measurement of $g_{s, i}^{(2)}>2$ is therefore a signature of non-classical correlations. However, in order to prove non-classicality without assumptions on the created state, the unconditional autocorrelation functions should be measured as well.

\subsection{A quantum light source compatible with $N$ d doped crystals}

Here we discuss a filtered SPDC source producing photons at $883 \mathrm{~nm}$ (signal) and $1338 \mathrm{~nm}$ (idler), which was developed to interface with a $\mathrm{Nd}^{3+}: \mathrm{Y}_{2} \mathrm{SiO}_{5}$ quantum memory operating at $883 \mathrm{~nm}$, having a memory bandwidth of $120 \mathrm{MHz}$. This particular source has been described in Refs [14, 80], while a similar source, slightly more broadband, of polarization-entangled photons for quantum storage was described in a more recent work [107].

The SPDC source was based on a periodically-poled potassium titanyl phosphate (PPKTP) crystal with an optical waveguide. The PPKTP crystal was pumped by a continuous-wave (cw) $532 \mathrm{~nm}$ laser, which is convenient since powerful and frequency stable single-mode Nd:YAG lasers exist at this wavelength. Since one photon should be resonant with the $883 \mathrm{~nm}$ transition in $\mathrm{Nd}^{3+}: \mathrm{Y}_{2} \mathrm{SiO}_{5}$, the choice of the 
pump laser imposed the wavelength of the idler photon to $1338 \mathrm{~nm}$, in the telecommunication O-band. In principle one could use another pump wavelength to produce an idler photon in the more conventional telecommunication C-band around 1550 nm.

Without external frequency filtering the source produced photons with a bandwidth of about $800 \mathrm{GHz}$, such that strong filtering of the photon pairs was necessary to match the memory bandwidth of $120 \mathrm{MHz}$. To this end a combination of diffraction gratings, optical cavities and a fibre-based filter was used. The gratings provided full-width at half-maximum (FWHM) bandwidths of 90 and $60 \mathrm{GHz}$ for the signal and idler photons, respectively. For the signal photon two etalons placed in series resulted in a single longitudinal mode with a bandwidth of $350 \mathrm{MHz}$. It should also be emphasized that the $6 \mathrm{GHz}$ wide inhomogeneous absorption profile of the $\mathrm{Nd}^{3+}: \mathrm{Y}_{2} \mathrm{SiO}_{5}$ crystal provided additional filtering, since the $120 \mathrm{MHz}$ comb was created within this absorption profile. For the idler photon a home-made narrow-band cavity filtered down the photons to a FWHM linewidth of $43 \mathrm{MHz}$. A single longitudinal mode of this cavity was selected by a fiber Bragg grating (FBG). The total transmission coefficients from the PPKTP waveguide to the single-mode fibers were $22 \%$ and $14 \%$ for signal and idler photons [80], respectively, including fiber coupling. The more recent version of this source [107] reach higher efficiencies, partly because high-efficiency volume Bragg gratings (VBGs) replaced the diffraction gratings.

Strong non-classical correlations between the signal and idler photons can only be obtained if the central frequencies of the filters on each mode satisfy the energy conservation of the SPDC processes. This is a non-trivial task when dealing with highly non-degenerate SPDC sources, particularly when one mode must be resonant with an external quantum memory. A solution to this problem was introduced in the work discussed here. A reference laser that is resonant with the signal filtering system and the quantum memory is injected into the SPDC source. This will create light at the idler wavelength through difference frequency generation (DFG), which

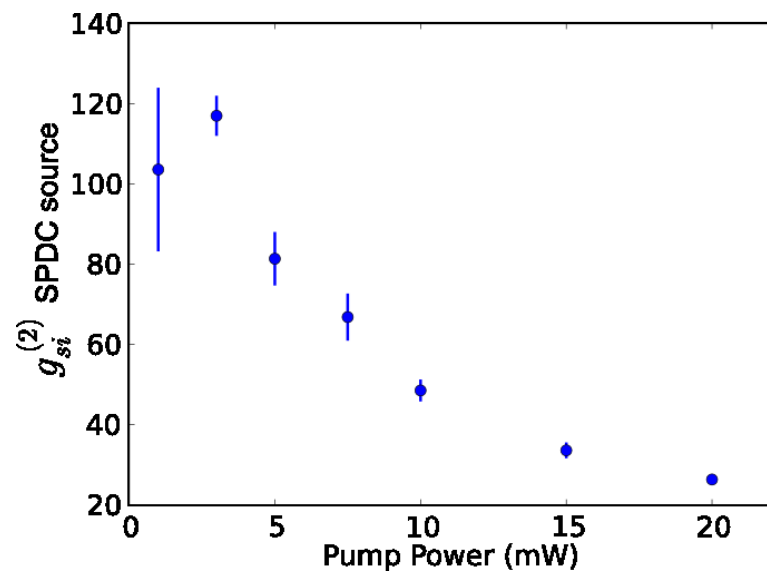

Fig. 2 Second-order crosscorrelation $g_{s, i}^{(2)}(\Delta \tau)$ function of the filtered SPDC source developed for Nd doped crystals, as a function of the power of the laser pumping the SPDC source. The detection integration window was $\Delta \tau=10 \mathrm{~ns}$. 
obey the necessary energy conservation. This can be used to adjust central frequency of the idler filtering system, alternatively one can also change the wavelength of the pump laser.

The filtered SPDC source can be characterized by the pair creation rate within the filtered modes (spectral brightness) and the second-order auto and cross correlation functions of the signal and idler modes. The intrinsic spectral brightness of the PPKTP waveguide was $6.3 \cdot 10^{3}$ pairs $/(\mathrm{mW} \cdot \mathrm{MHz} \cdot \mathrm{s})$ [80], which does not include the transmission through the filtering elements. This means that the probability $p$ to create a pair (in the limit $p \ll 1$ ) is then $p \approx 2.7 \cdot 10^{-3} / \mathrm{mW}$ per $10 \mathrm{~ns}$ within a $43 \mathrm{MHz}$ wide spectral window. The duration of the detection window should be set with respect to the photon pair coherence time, which in our case is dominated by the $43 \mathrm{MHz}$ filter on the idler side ( $7 \mathrm{~ns}$ coherence time). The spectral brightness of the source including the filtering elements and fiber coupling was $\approx 200$ pairs $/(\mathrm{mW}$ - $\mathrm{MHz} \cdot \mathrm{s}$ ). If we again assume a $43 \mathrm{MHz}$ filtered bandwidth we arrive at a final pair creation rate of 8600 pairs/s per $\mathrm{mW}$ pump power.

The second-order auto-correlation functions of both the idler and signal modes were characterized [80], resulting in $g_{i, i}^{(2)}(0)=1.9$ and $g_{s, s}^{(2)}(0)=1.8$. These values give effective mode numbers $K=1.1$ and $K=1.25$ for the idler and signal modes, which indicate single frequency modes on both the signal and idler side.

In Figure 2 we show the second-order cross-correlation function $g_{s, i}^{(2)}$ of the source as a function of pump power which exhibits the expected $1+1 / p$ behaviour [14]. Based on the intrinsic spectral brightness we expect a cross-correlation $g_{s, i}^{(2)} \approx 75$ for $P=5 \mathrm{~mW}$, which is very close to the measured value of about 80 . The cross-correlation function is larger than the classical upper bound of 2 , where one assumes $g_{i, i}^{(2)}(0)=g_{s, s}^{(2)}(0)=2$, for all pump powers, clearly demonstrating the strong non-classical correlations of the source.

\subsection{A quantum light source compatible with Pr doped crystals}

In the context of generating quantum light compatible with $\operatorname{Pr}^{3+}: \mathrm{Y}_{2} \mathrm{SO}_{5}$, one of the photons must be at $606 \mathrm{~nm}$, with a bandwidth smaller than $4 \mathrm{MHz}$. In addition, in order to use this source to generate entanglement between remote crystals, it is desirable to have the second photon of the pair at a telecom wavelength. An experiment showing these properties was reported in [84].

In order to meet these requirements, the following source was used. A pump laser at $426 \mathrm{~nm}$ was used to generate widely non degenerate photon pairs at $606 \mathrm{~nm}$ and $1436 \mathrm{~nm}$ in a periodically poled lithium niobate crystal. The crystal was placed in a bow tie optical cavity with a free spectral range of $400 \mathrm{MHz}$ and a finesse of around 200 for the signal and idler modes. A special coating was used to achieve high reflectivity $(99.99 \%)$ for three mirrors, and a specified reflectivity of $98.5 \%$ for the output coupler. The SPDC output is modified by the cavity modes. In the case of degenerate photons with the same polarization, the spectrum would be a convolution 


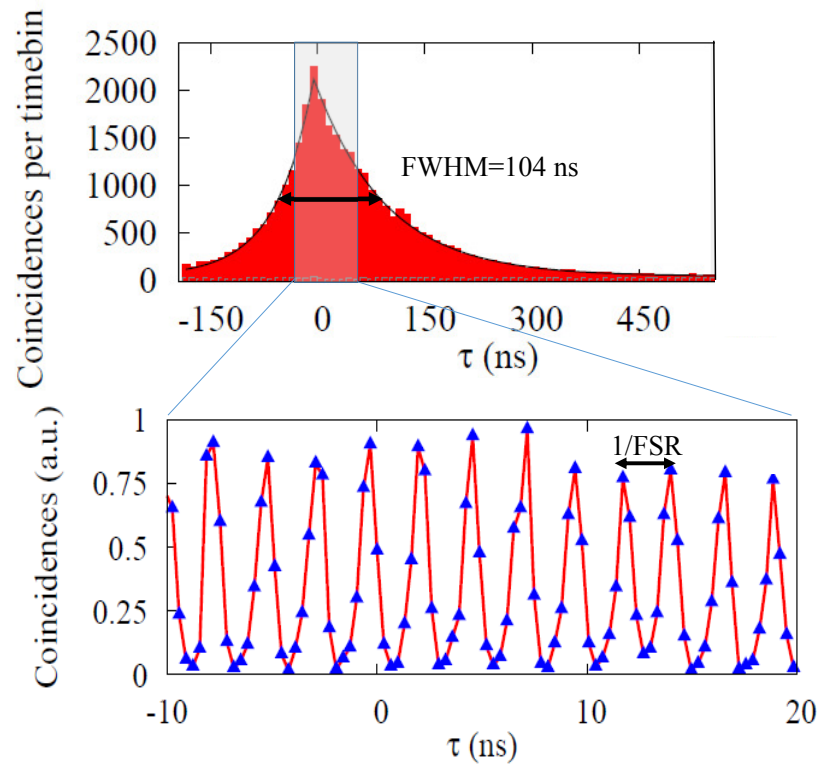

Fig. 3 Measured second-order cross-correlation $G_{s, i}^{(2)}(\tau)$ function (non normalized) of the cavity enhanced SPDC source developed for Pr doped crystals [84]. The FWHM correlation time is 104 ns. The zoom displays a higher temporal resolution, allowing to observe the oscillations in the $G_{s, i}^{(2)}(\tau)$, characteristic of multi spectral mode output. From this data, a number of four mode per cluster is inferred.

between the phase matching bandwidth and the cavity modes. However, in the non degenerate case, the signal and idler modes experience different dispersion characteristics in the crystal, which leads to slightly different free spectral ranges. Since both modes have to be resonant with the cavity to enable enhancement, only a subset of modes will be enhanced. These modes are grouped in so called clusters [92, 109]. The width and spacing of the clusters is determined by the dispersion properties of the crystals and the cavity geometry.

In the experiment of Ref. [84], the number of modes per cluster was inferred to be around 4, by looking at the oscillations in the $g_{s, i}^{(2)}(\tau)$ function and comparing to Eq. 9 (see Fig. 3). This number was also verified by direct measurement of the signal and idler fields with a narrow band filter cavity, scanned over the spectrum. The number of clusters was then inferred by measuring the first order correlation function of the idler field. Within the phase-matching bandwidth of the free space down conversion photons of around $\Gamma_{p m}=80 \mathrm{GHz}$, the spectrum of the photons leaving the cavity was finally inferred to be composed of one main cluster containing around four longitudinal modes, and two smaller side clusters separated by $45 \mathrm{GHz}$ from the main one and suppressed by around $80 \%$. For degenerate photons, the number of longitudinal modes would be given by $\Gamma_{p m} / F S R=200$ modes. The clustering effect 
therefore leads to a suppression of the number of modes of around 50. Within a longitudinal mode, the photons pair created featured a correlation time of $104 \mathrm{~ns}$ (see Fig. 3), the longest demonstrated so far with SPDC sources. From the decay of

the $G_{s, i}^{(2)}(\tau)$ (fitted with $\exp (-2 \pi \Delta v \tau)$ ), spectral-linewidth of $\Delta v$ of $1.7 \mathrm{MHz}$ and $2.9 \mathrm{MHz}$ were inferred for the idler field at $1436 \mathrm{~nm}$ and the signal field at $606 \mathrm{~nm}$, respectively. The asymmetry can be attributed to the different intra-cavity losses for the two wavelengths. A intrinsic spectral brightness of $8 \cdot 10^{3}$ pairs $/(\mathrm{mW} \cdot \mathrm{MHz}$ . s) was inferred, leading to $p \approx 6.4 \cdot 10^{-3} / \mathrm{mW}$ per $400 \mathrm{~ns}$ within a $2 \mathrm{MHz}$ wide spectral window. However due to large optical losses, the spectral brightness before the detectors (inside single mode fibers) was 11 pairs $/(\mathrm{mW} \cdot \mathrm{MHz} \cdot \mathrm{s})$. In a more recent version of the source, this number was increased to 190 pairs $/(\mathrm{mW} \cdot \mathrm{MHz}$. s).

This source still requires additional filtering for selecting a single frequency mode. However, the filtering requirement are considerably relaxed due to the low number of modes present in the spectrum. Side clusters can easily be removed by placing etalons with high transmission efficiency in the signal or idler modes. In order to select a single mode, a narrow band filter cavity with FSR $=16.8 \mathrm{GHz}$ and linewidth of $80 \mathrm{MHz}$ has been placed in the signal arm. In this configuration, the measured $G_{s, i}^{(2)}(\tau)$ was measured and no oscillation was observed, confirming that only one mode per cluster was present. The suppression of the side cluster could also be inferred first order autocorrelation.

\section{Quantum Light Storage experiments}

\subsection{Quantum entanglement storage in Nd:YSO crystals}

In 2008 the first $\mathrm{AFC}$ echo storage experiment in a $\mathrm{Nd}^{3+}$-doped $\mathrm{YVO}_{4}$ crystal at the single photon level was demonstrated [12], which was the starting point for considering storing true quantum states of light. To this end the filtered SPDC source described in section 5.2 was developed. The first $\mathrm{Nd} \mathrm{YVO}_{4}$ memory had a bandwidth of a few MHz, making the filtering of the SPDC source difficult. A more wideband quantum memory was therefore developed in a $\mathrm{Nd}^{3+}$-doped crystal $\mathrm{Y}_{2} \mathrm{SiO}_{5}$, using the ${ }^{4} \mathrm{I}_{9 / 2}-{ }^{4} \mathrm{~F}_{3 / 2}$ transition at $883 \mathrm{~nm}$ [74, 14]. This optical transition has an inhomogeneous broadening of about $6 \mathrm{GHz}$. The ground state was split into a Kramers spin doublet by applying a magnetic field of around 300 mTesla, producing a Zeeman split of about $11 \mathrm{GHz}$. The comb structure was created on one of the Zeeman transitions by performing spectral hole burning, i.e. ions were optically pumped into the other Zeeman spin state. In practice this was done by scanning the laser frequency of a narrow-band external-cavity diode laser (EDCL) with a acousto-optic modulator (AOM), while periodically switching off and on the light. The total bandwidth was $120 \mathrm{MHz}$, limited by the scan range of the AOM. The maximum efficiency at low storage times $1 / \Delta=25 \mathrm{~ns}$ was $20 \%$, close to the optimal value for the optical depth 
of the crystal. In Figure 4 we show an example of storage of $883 \mathrm{~nm}$ signal photons produced by the filtered SPDC source in the crystal [14]. The cross correlation function between the signal and idler modes after storing the signal mode clearly shows quantum correlations, for all storage times. This can be interpreted as storing a single photon at $883 \mathrm{~nm}$, heralded by the detection of an idler photon.

The second-order cross-correlation function $g_{s, i}^{(2)}(\Delta \tau)$ after storage of the signal photon is lower than that of the source, cf. Figure 2. In [80] it was shown that it was due to the delay introduced by the memory. Indeed, the signal photons released from the memory are superimposed with uncorrelated signal photons created later during the AFC echo emission, which are transmitted through the memory with a certain probability. This noise source depends on the ratio of the memory transmission to the memory efficiency [80]. A solution to this problem is to turn off the SPDC pump laser before the AFC echo, which was done in the quantum storage experiment in $\operatorname{Pr}^{3+}: \mathrm{Y}_{2} \mathrm{SiO}_{5}$ described below [16].

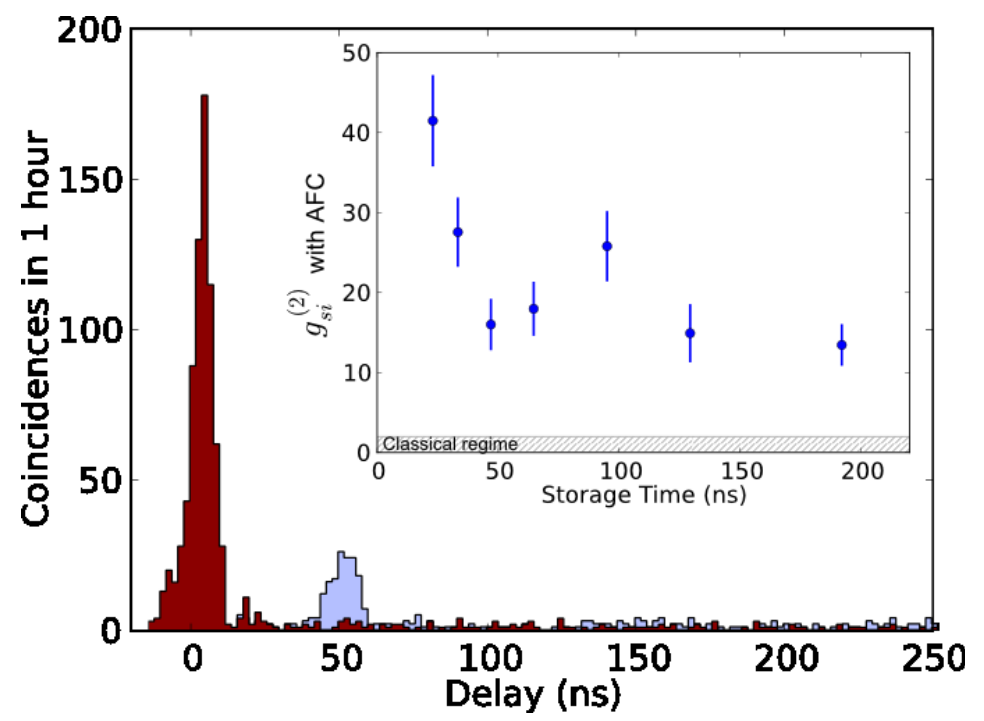

Fig. 4 Detection event histogram as a function of time. The peak at around zero time was recorded by making a large transparency hole in the $\mathrm{Nd}^{3+}: \mathrm{Y}_{2} \mathrm{SiO}_{5}$ absorption, therefore it represents the input mode. When creating an AFC with periodicity $\Delta=20 \mathrm{MHz}$, the expected AFC echo appears as a peak at around $50 \mathrm{~ns}$. The excellent signal-to-noise ratio indicates a strong quantum correlation between the idler photon $(1338 \mathrm{~nm})$ and the signal photon $(883 \mathrm{~nm})$, after the latter has been stored in the memory. The inset quantifies this by showing the second-order cross-correlation $g_{s, i}^{(2)}(\Delta \tau)$ function as a function of storage time $1 \Delta$. The $g_{s, i}^{(2)}(\Delta \tau)$ function is significantly larger than the classical limit of 2 , for all storage times. The detection integration window was $\Delta \tau=10 \mathrm{~ns}$.

The photons produced by a SPDC source pumped by a CW pump laser are entangled in energy-time [110], provided that the coherence time of the pump is significantly longer than the coherence time of the signal-idler pair. This condition is 


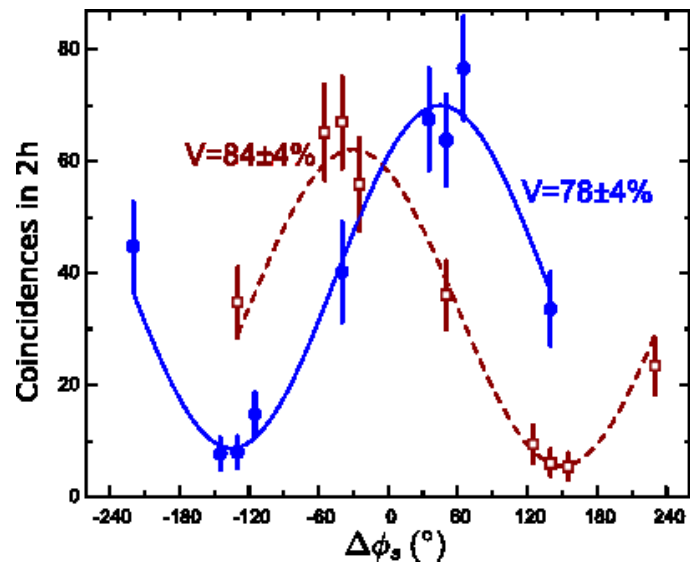

Fig. 5 Number of detected signal-idler coincidences as a function of the signal analyser setting $\Delta \phi_{s}$, for two settings $\Delta \phi_{i}$ of the idler analyser shown as square and circle symbols. The solid $(V=78 \pm 4 \%)$ and dashed $(V=84 \pm 4 \%)$ lines are fits to the circles and squares, respectively.

naturally met when using a single-mode frequency $\mathrm{CW}$ pump laser, as in this case. This energy-time entanglement can be revealed by making projective measurements on different time basis states, on both the signal and idler modes. Practically, one can place Mach-Zehnder (MZ) interferometers in each mode, each MZ having the same path difference $\Delta T$, which is known as a Franson-type set-up [110]. The two-photon interference fringes obtained when varying the phases in each interferometer reveals the energy-time entanglement. The coincidence rate varies like $V \cos \left(\Delta \phi_{s}+\Delta \phi_{s}\right)$, where $\phi_{s}$ and $\phi_{i}$ are the phase settings on the signal and idler interferometers and $V$ is the visibility. More formally the quantum entanglement can be detected by a violation of the Clauser-Horne-Shimony-Holt (CHSH) inequality [111], where the $\mathrm{CHSH}$ parameter $S$ is larger than 2 for any entangled state. The presence of entanglement can also be inferred from a fringe visibility larger than $1 / \sqrt{2} \approx 70.7 \%$.

In the experiment we describe here [14], a particular twist was introduced. While the idler photon was analyzed using a standard interferometer, the signal photon was analyzed inside the $\mathrm{Nd}^{3+}: \mathrm{Y}_{2} \mathrm{SiO}_{5}$ memory. We exploited the fact that more complicated absorption features than periodic combs can be created. Indeed, by creating an absorption structure that was the sum of two combs with different periodicities $\Delta_{1}$ and $\Delta_{2}$, we could create an effective unbalanced interferometer within the crystal. By setting $1 / \Delta_{1}-1 / \Delta_{2}=\Delta T$ we could analyze the entanglement in memory.

In Figure 5 we show examples of interference fringes as a function of $\Delta \phi_{s}$ for two values of $\Delta \phi_{i}$. Both visibilities are well above the limit $\approx 70.7 \%$, strongly indicating the presence of entanglement. By explicitly measuring a $\mathrm{CHSH}$ parameter of $S=$ $2.64 \pm 0.23$ the entanglement between the idler photon and the photon stored in the crystal was clearly demonstrated.

In parallel to the work described above, Saglamyurek et al. also demonstrated storage of a photon entangled with a another photon stored in and released from a rare-earth doped crystal [15]. In their experiment the entangled photons were produced by a bulk periodically poled lithium niobate (PPLN) crystal, which was pumped using a pico-second laser with high peak power. Time-bin entangled photons were produced by splitting the 16 ps pump pulse into two coherent pulses sep- 
arated by $1.4 \mathrm{~ns}$. The idler photon was at the telecom wavelength $1532 \mathrm{~nm}$, while the signal photon was at $795 \mathrm{~nm}$. The quantum memory was based on a atomic frequency comb created on the ${ }^{3} \mathrm{H}_{6}{ }^{3} \mathrm{H}_{4}$ transition at $795 \mathrm{~nm}$ in a Thulium-doped lithium niobate crystal $\left(\mathrm{Tm}^{3+}: \mathrm{LiNbO}_{3}\right)$. A particular feature of this experiment was the optical waveguide on the surface of the $\mathrm{Tm}^{3+}: \mathrm{LiNbO}_{3}$ chip. The waveguide technology is widespread in integrated optics and opens up interesting perspectives of combining the storage device with other optical elements. The AFC memory in this experiment was also very broadband, the AFC spanned a range of $5 \mathrm{GHz}$. This allowed storage of the short 16 ps photons produced by the filtered SPDC source. This also implies that the degree of filtering was less stringent than in the experiment described previously. A drawback of this material is, however, the short storage time and the low efficiency. The entanglement experiment discussed here demonstrated a storage time of $7 \mathrm{~ns}$, with an AFC echo efficiency of $2 \%$. The preservation of entanglement was proved by testing the Clauser-Horne-Shimony-Holt(CHSH) inequality, resulting in a CHSH parameter of $\mathrm{S}=2.25 \pm 0.06$.

In the next experiment that we will describe the goal was to entangle two $\mathrm{Nd}^{3+}: \mathrm{Y}_{2} \mathrm{SiO}_{5}$ crystals [80]. This could be done by storing each photon out of an entangled pair, but this requires that both photons are resonant with a memory. With only one photon in resonance with the $\mathrm{Nd}$ doped crystal at $883 \mathrm{~nm}$, the choice was made instead to store a path-entangled state of a single photon [112]. More specifically, this state can be created by sending a single photon state $|1\rangle$ through a balanced beam-splitter, which creates the state $1 / \sqrt{2}\left(|1\rangle_{A}|0\rangle_{B}+|0\rangle_{A}|1\rangle_{B}\right)$ of the spatial output modes A and B of the beam-splitter. This state is sometimes referred to as single-photon entanglement [112]. Now, entanglement between two memories can be realized by placing one memory in each path. This approach was first used by Choi et al. [113] to entangle two spatial modes in the same cloud of laser-cooled caesium atoms, where each mode was stored in the cloud using EIT. In the experiment described here, this this approach was used to entangle the modes of two physically distinct crystals and the modes where stored using the AFC scheme [80]. The single photon state was produced by detecting the idler photon from the filtered SPDC source, which creates a state very close to a single photon in the signal mode (a heralded single-photon source).

To characterize the single-photon entanglement one can read out the memories and then performing measurements on the photonic state, which provides a lower bound of the entanglement present while storing the two modes. To detect the entanglement we used the tomographic approach developed by Chou et al. [114], in which the entanglement is quantified through a single parameter, the concurrence $C$. The concurrence is positive $C>0$ for an entangled state, more specifically a separable state gives $C=0$ and a maximally entangled state $C=1$. To compute the concurrence one needs to measure the probability of finding exactly one signal photon in any of the two modes, $p_{01}$ and $p_{10}$, and the probability of finding one signal photon in each path, $p_{11}$. This requires two- and three-fold coincidence measurements, respectively, since one also needs to detect the heralding idler photon. In addition one needs to measure the one-photon visibility $V$ associated with the two paths A and B. The concurrence can then be calculated with the formula: 
$C=V\left(p_{10}+p_{01}\right)-\sqrt{2 p_{00} p_{11}}$ [114]. By performing all the necessary measurements for a relatively high pump power of $16 \mathrm{~mW}$, we reached a concurrence of $C=6.3 \pm 3.8 \cdot 10^{-5}$ indicating presence of entanglement [80]. The low concurrence was essentially due to losses, since the detection probabilities include all propagation losses, memory efficiency and detection probabilities. Alternatively one can use an approach where the $p_{11}$ probability is inferred from the cross-correlation function $g_{s, i}^{(2)}$, which is a much more time efficient experiment as it uses only two-fold detections. Using this approach the presence of entanglement at the pump power of $16 \mathrm{~mW}$ could be confirmed, and the concurrence was measured for a range of lower pump powers where the direct method turned out to be too time consuming due to the rarity of three-fold coincidences. The concurrence reached about $C=1.1 \pm 0.1 \cdot 10^{-4}$ for the lowest pump power of $1 \mathrm{~mW}$, showing the presence of entanglement of the stored modes in the two $\mathrm{Nd}^{3+}: \mathrm{Y}_{2} \mathrm{SiO}_{5}$ crystals.

\subsection{Quantum Storage of heralded single photon in a $\mathrm{Pr}^{3+}: \mathrm{Y}_{2} \mathrm{SiO}_{5}$ crystal}

As mentioned previously, $\operatorname{Pr}$ doped solids have demonstrated exceptional properties for light storage experiments, including long storage times [83, 40, 41] and high efficiencies [13, 69]. Despite these very promising properties, there is currently only one demonstration of storage of quantum light in this system. In this section, we describe in more detail this demonstration, initially reported in [16].

The ultra-narrowband photon pair source described in section 5.3 can be used to generate heralded single photons compatible with the Praseodymium doped crystal. For sufficiently high correlations between the two fields, the detection of an idler telecom photon will indeed project the signal mode in a single photon Fock state.

These heralded single photons have been stored in the $\mathrm{Pr}^{3+}: \mathrm{Y}_{2} \mathrm{SiO}_{5}$ as collective optical atomic excitations using the AFC echo scheme. The storage device is a 3 mm thick $\mathrm{Y}_{2} \mathrm{SiO}_{5}$ sample doped with a $\mathrm{Pr}^{3+}$ concentration of $0.05 \%$. The relevant optical transition connects the ${ }^{3} H_{4}$ ground state to the ${ }^{1} D_{2}$ excited state at a wavelength of $605.977 \mathrm{~nm}$ and features a measured absorption coefficient of $23 \mathrm{~cm}^{-1}$, and an inhomogeneous linewidth of $5 \mathrm{GHz}$. At zero magnetic field, the ground state and excited states manifolds are split in 3 metastable states, denoted $\pm 1 / 2_{k}, \pm 3 / 2_{k}$ and $\pm 5 / 2_{k}$ (see inset of Fig. 6), where $k=g$, e denotes the ground or the excited state. The spacing between the hyperfine states is of the order of a few MHz. This gives an upper limit for the bandwidth as already mentioned. But, since the separation between ground states is much smaller than the inhomogeneous broadening, it also creates a complication for isolating a single class of atoms. A laser sent in the crystal can indeed be resonant with up to 9 distinct classes of atoms, within the inhomogeneous broadening. To select a single class of atoms, the optical pumping scheme first demonstrated by Nilsson et al was used [115]. After selecting one class of atoms, an atomic frequency comb is created on the $\pm 1 / 2_{g} \rightarrow \pm 3 / 2_{e}$ transition (see Fig. 6 for an example of AFC). 


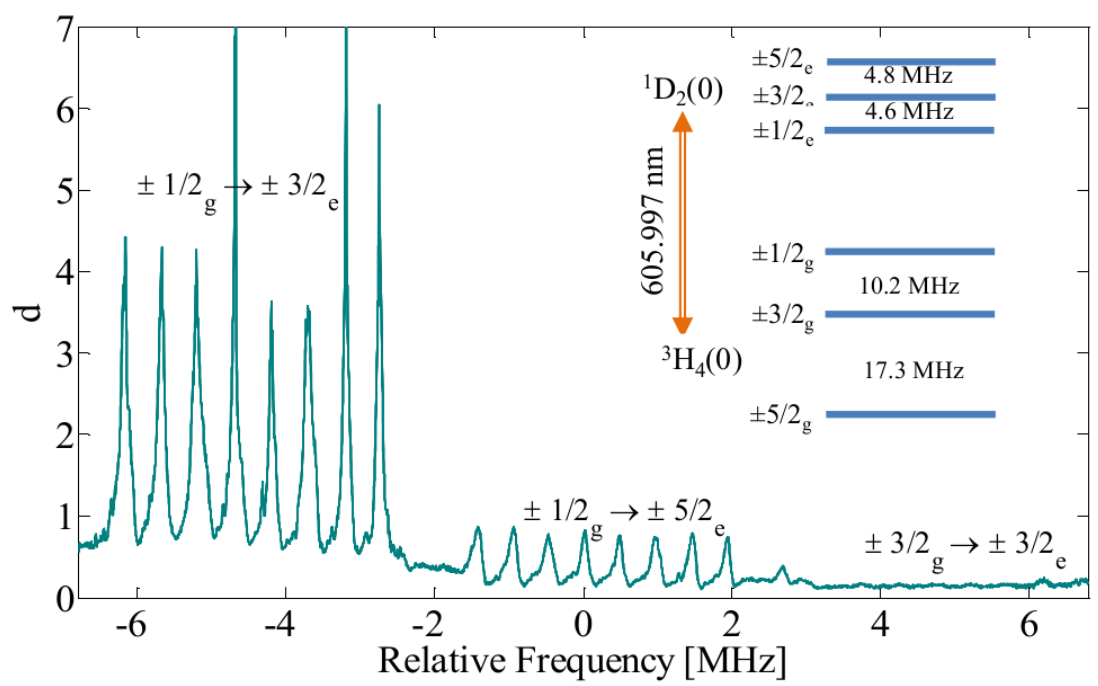

Fig. 6 Exemple of an atomic frequency comb in $\mathrm{Pr}^{3+}: \mathrm{Y}_{2} \mathrm{SiO}_{5}$. The optical depth $(d)$ is plotted as a function of the relative frequency. The input and output photons are resonant with the $\pm 1 / 2_{g} \rightarrow$ $\pm 3 / 2_{e}$ transition. Inset : Relevant energy level scheme of the $\mathrm{Pr}^{3+}: \mathrm{Y}_{2} \mathrm{SiO}_{5}$ crystal, with 3 hyperfine ground states and 3 excited states.

One important issue to couple photons to the solid state atomic ensemble is that the frequency of the photons must be stable within a few hundred $\mathrm{kHz}$. This can be insured by implementing a feedback lock system using the laser used to prepare the atomic frequency comb. One the one hand, the length of the cavity is locked on the laser with a Pound-Drever-Hall scheme. This insures that at least one spectral mode emitted by the cavity enhanced source is resonant with the crystal absorption. On the other hand, the required double resonance for signal and idler modes is insured by adjusting the frequency of the pump laser using a classical signal at the idler wavelength created by difference frequency generation between the pump laser at $426 \mathrm{~nm}$ and the laser at $606 \mathrm{~nm}$.

In the experiment, a single longitudinal mode was selected for the idler mode thanks to a Fabry Perot filter cavity. For the heralded single photon, the crystal itself was used as a filter to prevent the modes non resonant with the AFC to reach the detector. The probability to have a single photon in the signal mode before the cryostat conditioned on a detection in the idler mode (called the heralding efficiency $\eta_{H}$ ) was around a few percents in this experiment, limited by dark counts in the idler detector, noise in the idler mode, cavity escape efficiency and optical losses in the signal mode from the cavity to the crystal. 
The heralded single photon at $606 \mathrm{~nm}$ was first characterized by measuring the second order correlation $g_{s, i}^{(2)}(\Delta \tau)$ function by sending it through the crystal where a $12 \mathrm{MHz}$ wide transparency window was created. In Fig. $7 \mathrm{p}$, the values of $g_{s, i}^{(2)}(\Delta \tau)$ for the incoming photons are plotted as a function of the pump power. The value of $g_{s, i}^{(2)}(\Delta \tau)$ increases when the pump power decreases, as expected for a two-mode squeezed state. The non classicality of the input photon was also demonstrated by violating a Cauchy-Schwarz inequality. The detected coincidence count rate was around $0.8 \mathrm{~Hz}$ per mW of pump power. By correcting for known optical losses and detection efficiencies, a creation rate outside the cavity of $2.8 \mathrm{kHz} / \mathrm{mW}$ was inferred.
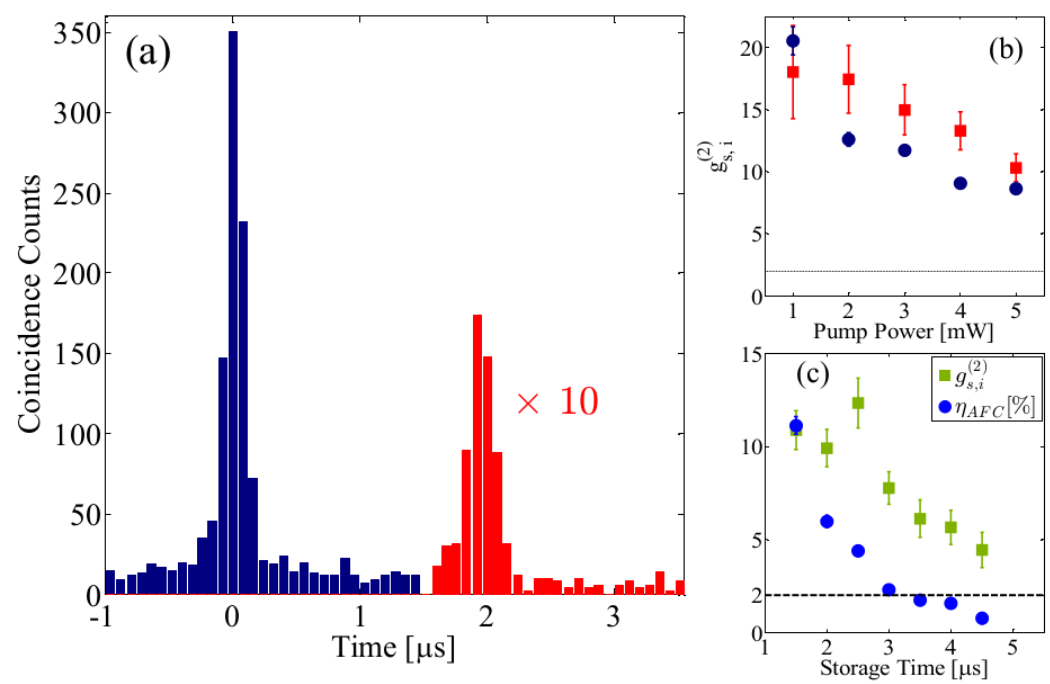

Fig. 7 Results of heralded single photon storage in a $\mathrm{Pr}^{3+}: \mathrm{Y}_{2} \mathrm{SiO}_{5}$ crystal [16]. (a) $G_{s, i}^{(2)}(t)$ histogram without (blue) and with (red) AFC. The preprogrammed storage time is $2 \mu$ s and the power of the $426.2 \mathrm{~nm}$ pump is $2 \mathrm{~mW}$. (b) The $g_{s, i}^{(2)}$ values as a function of the pump power for the AFC echo (plain squares) are compared to those for the input photons (plain circles). The dotted line corresponds to the classical limit $g_{s, i}^{(2)}=2$ for two-mode squeezed states. (c) Storage and retrieval efficiency (blue circle) and $g_{s, i}^{(2)}$ (green squares) as a function of the storage time. For (b) and (c) the data are evaluated for a detection window $\Delta \tau=400 \mathrm{~ns}$ and the error bars are evaluated from the raw number of counts assuming Poissonian statistics.

After having confirmed the non classicality of the input light, the heralded single photon was stored in and retrieved from the crystal using atomic frequency comb. In order to avoid the spurious noise effect described in section 6.1, the photon pair 
source pump light was switched off after detection of an idler photon. The efficiency of the storage and retrieval process was measured to be up to $10 \%$ for short storage times. The second order cross correlation is measured for the stored and retrieved photon as can be seen in Fig. 77. The values of $g_{s, i}^{(2)}$ are also plotted as a function of the pump power in Fig. 7p. Surprisingly, the values of $g_{s, i}^{(2)}$ after retrieval are higher than the one for the input photons, for a big range of pump powers. This effect has been attributed to the fact that the memory act as a filter for broadband noise emitted by the photon pair source. Since the pump light is switched off after the detection of an idler photon, the atomic frequency comb delays the signal photon in a noise free region, which therefore increases the signal to noise ratio and the $g_{s, i}^{(2)}(\Delta \tau)$ [16]. This effect highlights that under certain conditions, quantum memories can act as purifiers by storing only the signal and not the noise. It has been observed also in other experiments [116, 73].

The storage time in the crystal can be chosen by tuning the comb periodicity $\Delta$. In theory, the minimal $\Delta$ achievable is given by $2 \gamma_{h} F$, where $\gamma_{h}$ is the homogeneous linewidth of the optical transition and $F$ the finesse of the comb. However, in practice, several effects will limit the achievable $\Delta$, including power broadening, finite laser linewidth, spin inhomogeneous broadening, crystal vibrations in closed loop cryostats, etc. In the present experiment, non classical correlations between heralding photon and stored and retrieved heralded photons have been observed until a storage time of $4.5 \mu$ s (see Fig. 7k). This is more than 20 times longer than previous realizations [14, 15] and would allow entanglement between crystals separated by $\mathrm{km}$ long distance. The storage and retrieval efficiency dropped by a factor around 10 between 1.5 and 4.5 us (see Fig. 7 7 F). This is mainly due to the fact that the finesse could not be kept constant when decreasing $\Delta$, because of the minimum achievable width of an absorption peak, due to the limitations mentioned above. Longer storage times in the excited state of up to 10 us have recently been obtained with weak coherent states in $\operatorname{Pr}^{3+}: \mathrm{Y}_{2} \mathrm{SiO}_{5}$ [73]. Note also that excited state storage times of up to 30 us have been obtained using bright pulses storage in a $\mathrm{Eu}^{3+}: \mathrm{Y}_{2} \mathrm{SiO}_{5}$ crystal [70].

\section{Prospects for spin-wave storage with quantum light}

In order to increase the storage time and to achieve an AFC spin wave memory with on demand read-out, it has been proposed to transfer collective optical atomic excitations to collective spin excitations (or spin waves), using control fields (see section 3). Proof of principle experiments have been realized in the classical regime for storage of strong pulses [117, 118, 70] and we discuss in this section the prospects to extend this experiments to quantum light. As mentioned in section 3 spin wave storage requires materials with at least 3 long lived ground state levels. The best known materials with the required properties are $\mathrm{Pr}^{3+}$ and $\mathrm{Eu}^{3+}$ doped crystals. 
The main experimental challenge for reaching the quantum regime with spinwave storage is to suppress the noise generated by the control pulses, which are very close in frequency from the single photon output (e.g. from 10 to $17 \mathrm{MHz}$ in $\mathrm{Pr}^{3+}: \mathrm{Y}_{2} \mathrm{SiO}_{5}$ and from 35 to $120 \mathrm{MHz}$ in $\mathrm{Eu}^{3+}: \mathrm{Y}_{2} \mathrm{SiO}_{5}$ ). In that respect, one advantage of the AFC scheme is that the control pulses are temporally separated from the single photon output, which allow the use of temporal filtering. However, the control pulses also create transient phenomena inside the crystal due to e.g. the interaction with unwanted residual population in the storage state because of imperfect optical pumping. This can give rise to free induction decay and fluorescence, which lead to noise emission simultaneous with the single photon emission. The suppression of this noise requires narrow spectral filtering, which can be realized using e.g. a narrow-band optical cavity or a crystal filter [119, 120]. An interesting figure of merit in the context of single-photon-level spin-wave storage, taking into account the noise generated and the storage and retrieval efficiency, is the mean number of input photons to achieve a signal-to-noise ratio (SNR) of 1 in the output mode, denoted as $\mu_{1}$. In 2013, an experiment in $\mathrm{Eu}^{3+}: \mathrm{Y}_{2} \mathrm{SiO}_{5}$ achieved $\mu_{1}=2.5$, using a Fabry-Perot narrowband filter cavity [121]. Very recently, a lower value of $\mu_{1}=0.1$ has been demonstrated in $\mathrm{Eu}^{3+}: \mathrm{Y}_{2} \mathrm{SiO}_{5}$ [122]. A low value of $\mu_{1}=0.07$ has also been shown in $\mathrm{Pr}^{3+}: \mathrm{Y}_{2} \mathrm{SiO}_{5}$ [123], using a reconfigurable transparency window in an another $\mathrm{Pr}^{3+}: \mathrm{Y}_{2} \mathrm{SiO}_{5}$ crystal as spectral filter. High fidelity storage of time-bin qubits encoded in weak-coherent states at the single photon level was also demonstrated [123], allowing the spin-wave memory to operate in the quantum regime. In order to achieve high fidelity storage of quantum light, it is important to obtain $\mu_{1} \ll 1$. This is because in practice, because of various optical losses, it is very difficult to obtain a single photon in front of the memory, with efficiency approaching unity. To achieve quantum storage with high SNR using the photon pair sources presented in section 5. the condition $\mu_{1} \ll \eta_{H}$ must be fulfilled. Such an experiment has so far not been demonstrated.

Beyond the challenge of storing a single photon as a single spin-wave excitation, another important challenge is to increase the spin-wave storage time in this single-excitation regime. The spin-wave memory in a rare-earth-ion doped crystal is limited by the inhomogeneous spin linewidth [117]. This limitation can be lifted by utilizing spin-echo techniques to rephase the inhomogeneous spin dephasing, in which case the storage time is limited by the spin coherence time. But one can push the storage time still further by implementing dynamical decoupling sequences to reach storage times beyond $1 \mathrm{~s}$, a technique that has been successfully implemented in EIT storage experiments of strong optical pulses in rare-earth-ion doped crystals [83, 41]. However, the use of spin echo tecyhniques for extending the storage time of ensemble-based quantum memories introduces a new potential source of noise. The challenge lies in avoiding to populate the $|s\rangle$ state with too many atoms, which would lead to spontaneous emission noise in the output when reading out the single spin excitation. At first this might appear to be almost impossible for a single excitation in $|s\rangle$ [124], but it was shown later that the strong collective emission into a particular spatial mode of the stored single excitation provides a very effective spatial filtering of the spontaneous emission noise [125]. The spin-echo technique 
must be very efficient, however, to avoid this noise, and it remains to be seen if the storage fidelity of a single photon can be high enough when applying spin echo techniques. In a very recent experiment [122] it was shown that spin echo techniques could indeed be used when manipulating an average spin excitation of around 1, in an ensemble composed of $10^{10} \mathrm{Eu}^{3+}$ ions, without adding significant noise to the optical read out of the memory. This was made possible by using a robust and error compensating spin echo sequence which limited the population introduced to the $|s\rangle$ state to well below $1 \%$. In this way a $\mu_{1}$ parameter of around 0.3 could be maintained for up to $1 \mathrm{~ms}$ of spin-wave storage time, showing that the noise level should in principle allow quantum state storage on a milliseconds time scale. One future goal of this research is to implement dynamical decoupling sequences in order to further extend the spin-wave storage time.

\section{Outlook}

Progress to harness the interaction between quantum light and atomic ensembles in a solid state environment has been fast in recent years. However, the full capabilities of these materials have not yet been exploited in the quantum regime and several challenges remain. For example, an experiment demonstrating simultaneously high efficiency and long storage time of quantum light has not been demonstrated yet. The quantum networking capabilities also need to be improved. Current research directions include work towards the realization of long lived heralded entanglement between remote solid state multimode quantum memories [11]. Such an experiment would pave the way to functional elementary segments of quantum repeaters with multiplexed entanglement generation. These applications would strongly benefit from the realization of a solid state photon pair source with embedded memory [87]. Another research direction actively pursued is to increase the spectral multiplexing capabilities, together with selective frequency read-out [62, 126]. Another promising research direction is the integration of these quantum memories with micro and nanostructures, which would open many interesting opportunities in terms of miniaturization, scalability and integration other optical elements such as quantum light sources and single photon detectors. The coupling of rare-earth ions with nanophotonic structures like photonic crystal waveguides or cavities would also provide increased light-matter interaction and potentially lead to cavity QED experiments with a low number of rare-earth ions [127]. This may also facilitate the detection and manipulation of single rare-earth ions that could be used as quantum bits [128, 129]. Finally, rare-earth ion doped crystals can be used as spin ensemble that can be coupled to superconductive cavities [130, 131], with the long term goal of connecting superconducting qubits and optical photons.

Acknowledgements The results described here has been obtained during several years of work by the teams at the University of Geneva and ICFO. The authors would like to particularly acknowledge the contributions by their co-authors of the work reviewed here, Christoph Clausen, Imam Us- 
mani, Félix Bussières, Nicolas Sangouard, Nicolas Gisin, Daniel Rielander, Kutlu Kutluer, Mustafa Gundogan, Patrick Ledingham, Margherita Mazzera, Julia Fekete and Matteo Cristiani.

\section{References}

1. Hammerer, K., Sørensen, A. S., and Polzik, E. S. Quantum interface between light and atomic ensembles. Rev. Mod. Phys. 82, 1041-1093 (2010).

2. Lvovsky, A. I., Sanders, B. C., and Tittel, W. Optical quantum memory. Nat Photon 3, 706-714 (2009).

3. Simon, C. et al. Quantum memories. The European Physical Journal D - Atomic, Molecular, Optical and Plasma Physics 58, 1-22 (2010-05-01).

4. Bussières, F. et al. Prospective applications of optical quantum memories. Journal of Modern Optics 60, 1519-1537 (2013).

5. Nunn, J. et al. Enhancing multiphoton rates with quantum memories. Phys. Rev. Lett. 110, 133601- (2013).

6. Kimble, H. J. The quantum internet. Nature 453, 1023-1030 (2008).

7. Briegel, H.-J., Dür, W., Cirac, J. I., and Zoller, P. Quantum repeaters: The role of imperfect local operations in quantum communication. Phys. Rev. Lett. 81, 5932-5935 (1998).

8. Duan, L.-M., Lukin, M. D., Cirac, J. I., and Zoller, P. Long-distance quantum communication with atomic ensembles and linear optics. Nature 414, 413-418 (2001).

9. Sangouard, N., Simon, C., de Riedmatten, H., and Gisin, N. Quantum repeaters based on atomic ensembles and linear optics. Rev. Mod. Phys. 83, 33-80 (2011).

10. Specht, H. P. et al. A single-atom quantum memory. Nature 473, 190-193 (2011).

11. Simon, C. et al. Quantum repeaters with photon pair sources and multimode memories. Phys. Rev. Lett. 98, 190503-4 (2007).

12. de Riedmatten, H., Afzelius, M., Staudt, M. U., Simon, C., and Gisin, N. A solid-state lightmatter interface at the single-photon level. Nature 456, 773-777 (2008).

13. Hedges, M. P., Longdell, J. J., Li, Y., and Sellars, M. J. Efficient quantum memory for light. Nature 465, 1052-1056 (2010).

14. Clausen, C. et al. Quantum storage of photonic entanglement in a crystal. Nature 469, 508-511 (2011).

15. Saglamyurek, E. et al. Broadband waveguide quantum memory for entangled photons. $\mathrm{Na}$ ture 469, 512-515 (2011).

16. Rieländer, D. et al. Quantum storage of heralded single photons in a praseodymium-doped crystal. Phys. Rev. Lett. 112, 040504- (2014).

17. Togan, E. et al. Quantum entanglement between an optical photon and a solid-state spin qubit. Nature 466, 730-734 (2010).

18. Bernien, H. et al. Heralded entanglement between solid-state qubits separated by three metres. Nature 497, 86-90 (2013).

19. K.C., L. et al. Macroscopic non-classical states and terahertz quantum processing in roomtemperature diamond. Nat Photon 6, 41-44 (2012).

20. England, D. G., Bustard, P. J., Nunn, J., Lausten, R., and Sussman, B. J. From photons to phonons and back: A THz optical memory in diamond. Phys. Rev. Lett. 111, 243601- (2013).

21. De Greve, K. et al. Quantum-dot spin-photon entanglement via frequency downconversion to telecom wavelength. Nature 491, 421-425 (2012).

22. Bao, X.-H. et al. Efficient and long-lived quantum memory with cold atoms inside a ring cavity. Nat Phys 8, 517-521 (2012).

23. Macfarlane, R. M. High-resolution laser spectroscopy of rare-earth doped insulators: a personal perspective. J. Lumin. 100, 1-20 (2002).

24. Macfarlane, R. and Shelby, R. Coherent Transients And Holeburning Spectroscopy In Rare Earth Ions In Solids; Spectroscopy Of Crystals Containing Rare Earth Ions. Elsevier Science Publishers, Amsterdam, Netherlands, (1987). 
25. Liu, G. and Jacquier, B. (eds.). Spectroscopic Properties of Rare Earths in Optical Materials. Springer-Verlag, Berlin Heidelberg, (2005).

26. Macfarlane, R. M. Inhomogeneous broadening of spectral lines in doped insulators. J. Lumin. 45, 1-5 (1990).

27. Macfarlane, R. M. Optical stark spectroscopy of solids. Journal of Luminescence 125, 156174 (2007).

28. Tittel, W. et al. Photon-echo quantum memory in solid state systems. Laser \& Photon. Rev. 4, 244-267 (2010)

29. Thiel, C., Böttger, T., and Cone, R. Rare-earth-doped materials for applications in quantum information storage and signal processing. Journal of Luminescence 131, 353 - 361 (2011).

30. Stoneham, A. M. Shapes of inhomogeneously broadened resonance lines in solids. Rev. Mod. Phys. 41, 82-108 (1969).

31. Sellars, M., Fraval, E., and Longdell, J. Investigation of static electric dipole-dipole coupling induced optical inhomogeneous broadening in $\mathrm{Eu}^{3+}: \mathrm{Y}_{2} \mathrm{SiO}_{5}$. Journal of Luminescence 107, 150-154 (2004).

32. Böttger, T., Thiel, C. W., Sun, Y., and Cone, R. L. Optical decoherence and spectral diffusion at $1.5 \mu \mathrm{m}$ in $\mathrm{Er}^{3+}: \mathrm{Y}_{2} \mathrm{SiO}_{5}$ versus magnetic field, temperature, and $\mathrm{Er}^{3+}$ concentration. Phys. Rev. B 73, 075101 (2006).

33. Macfarlane, R. M., Meltzer, R. S., and Malkin, B. Optical measurement of the isotope shifts and hyperfine and superhyperfine interactions of nd in the solid state. Phys. Rev. B 58, 5692 (1998).

34. Sun, Y., Thiel, C. W., Cone, R. L., Equall, R. W., and Hutcheson, R. L. Recent progress in developing new rare earth materials for hole burning and coherent transient applications. $J$. Lumin. 98, 281-287 (2002).

35. Macfarlane, R. and Shelby, R. Sub-kilohertz optical linewidths of the ${ }^{7} \mathrm{f}_{0}-{ }^{5} \mathrm{~d}_{0}$ transition in $\mathrm{Y}_{2} \mathrm{O}_{3}: \mathrm{Eu}^{3+}$. Optics Communications 39, 169-171 (1981).

36. Yano, R., Mitsunaga, M., and Uesugi, N. Ultralong optical dephasing in $\mathrm{Eu}^{3+}: \mathrm{Y}_{2} \mathrm{SiO}_{5}$. Opt. Lett. 16, 1884- (1991).

37. Fraval, E., Sellars, M. J., and Longdell, J. J. Method of extending hyperfine coherence times in $\mathrm{Pr}^{3+}: \mathrm{Y}_{2} \mathrm{SiO}_{5}$. Phys. Rev. Lett. 92, 077601-4 (2004).

38. Fraval, E., Sellars, M. J., and Longdell, J. J. Dynamic decoherence control of a solid-state nuclear-quadrupole qubit. Phys. Rev. Lett. 95, 030506 (2005).

39. Pascual-Winter, M. F., Tongning, R.-C., Chanelière, T., and Le Gouët, J.-L. Spin coherence lifetime extension in $\mathrm{Tm}^{3+}$ :YAG through dynamical decoupling. Phys. Rev. B 86, 184301(2012).

40. Lovric, M., Suter, D., Ferrier, A., and Goldner, P. Faithful solid state optical memory with dynamically decoupled spin wave storage. Phys. Rev. Lett. 111, 020503-(2013).

41. Heinze, G., Hubrich, C., and Halfmann, T. Stopped light and image storage by electromagnetically induced transparency up to the regime of one minute. Phys. Rev. Lett. 111, 033601(2013).

42. Zhong, M., Hedges, M. P., Ahlefeldt, R. L. and Bartholomew, J. G., and Beavan, S. E. , Wittig, S. M., Longdell, J.J., Sellars, M. J. Optically addressable nuclear spins in a solid with a six-hour coherence time. Nature. 517, 177- (2015).

43. McAuslan, D. L., Longdell, J. J., and Sellars, M. J. Strong-coupling cavity qed using rareearth-metal-ion dopants in monolithic resonators: What you can do with a weak oscillator. Phys. Rev. A 80, 062307- (2009).

44. Sun, Y., Böttger, T., Thiel, C. W., and Cone, R. L. Magnetic g tensors for the ${ }^{4} \mathrm{I}_{15 / 2}$ and ${ }^{4} \mathrm{I}_{13 / 2}$ states of $\mathrm{Er}^{3+}: \mathrm{Y}_{2} \mathrm{SiO}_{5}$. Phys. Rev. B 77, 085124-10 (2008).

45. Guillot-Noël, O. et al. Hyperfine interaction of $\mathrm{Er}^{3+}$ ions in $\mathrm{Y}_{2} \mathrm{SiO}_{5}$ : An electron paramagnetic resonance spectroscopy study. Phys. Rev. B 74, 214409-8 (2006).

46. Ruggiero, J., Gouët, J.-L. L., Simon, C., and Chanelière, T. Why the two-pulse photon echo is not a good quantum memory protocol. Phys. Rev. A 79, 053851-8 (2009).

47. Moiseev, S. A. and Kröll, S. Complete reconstruction of the quantum state of a single-photon wave packet absorbed by a Doppler-broadened transition. Phys. Rev. Lett. 87, 173601-4 (2001). 
48. Nilsson, M. and Kröll, S. Solid state quantum memory using complete absorption and reemission of photons by tailored and externally controlled inhomogeneous absorption profiles. Optics Communications 247, 393-403 (2005).

49. Kraus, B. et al. Quantum memory for nonstationary light fields based on controlled reversible inhomogeneous broadening. Phys. Rev. A 73, 020302-4 (2006).

50. Alexander, A. L., Longdell, J. J., Sellars, M. J., and Manson, N. B. Photon echoes produced by switching electric fields. Phys. Rev. Lett. 96, 043602-4 (2006).

51. Hétet, G. et al. Photon echoes generated by reversing magnetic field gradients in a rubidium vapor. Opt. Lett. 33, 2323-2325 (2008).

52. Hétet, G., Longdell, J. J., Alexander, A. L., Lam, P. K., and Sellars, M. J. Electro-optic quantum memory for light using two-level atoms. Phys. Rev. Lett. 100, 023601-4 (2008).

53. Lauritzen, B. et al. Telecommunication-wavelength solid-state memory at the single photon level. Phys. Rev. Lett. 104, 080502- (2010).

54. Chanelière, T. et al. $\mathrm{Tm}^{3+}: \mathrm{Y}_{2} \mathrm{O}_{3}$ investigated for a quantum light storage application. Phys. Rev. B 77, 245127 (2008).

55. Afzelius, M., Simon, C., de Riedmatten, H., and Gisin, N. Multimode quantum memory based on atomic frequency combs. Phys. Rev. A 79, 052329-9 (2009).

56. Nunn, J. et al. Multimode memories in atomic ensembles. Phys. Rev. Lett. 101, 260502-4 (2008).

57. Dicke, R. H. Coherence in spontaneous radiation processes. Phys. Rev. 93, 99-110 (1954).

58. Mossberg, T., Flusberg, A., Kachru, R., and Hartmann, S. R. Total scattering cross section for Na on He measured by stimulated photon echoes. Phys. Rev. Lett. 42, 1665-1669 (1979).

59. Carlson, N. W., Bai, Y. S., Babbitt, W. R., and Mossberg, T. W. Temporally programmed free-induction decay. Phys. Rev. A 30, 1572-1574 (1984).

60. Mitsunaga, M., Yano, R., and Uesugi, N. Spectrally programmed stimulated photon echo. Opt. Lett. 16, 264-266 (1991).

61. Bonarota, M., Gouët, J.-L. L., Moiseev, S. A., and Chanelière, T. Atomic frequency comb storage as a slow-light effect. Journal of Physics B: Atomic, Molecular and Optical Physics 45, 124002 (2012).

62. Sinclair, N. et al. Spectral multiplexing for scalable quantum photonics using an atomic frequency comb quantum memory and feed-forward control. Phys. Rev. Lett. 113, 053603(2014).

63. Moiseev, S. and Noskov, M. The possibilities of the quantum memory realization for short pulses of light in the photon echo technique. Laser Phys. Lett. 1, 303-310 (2004).

64. Sangouard, N., Simon, C., Afzelius, M., and Gisin, N. Analysis of a quantum memory for photons based on controlled reversible inhomogeneous broadening. Phys. Rev. A $\mathbf{7 5}$, 032327-8 (2007).

65. Chanelière, T., Ruggiero, J., Bonarota, M., Afzelius, M., and Gout, J.-L. L. Efficient light storage in a crystal using an atomic frequency comb. New Journal of Physics 12, 023025(2010).

66. Bonarota, M., Ruggiero, J., Le Gouët, J.-L., and Chanelière, T. Efficiency optimization for atomic frequency comb storage. Phys. Rev. A 81, 033803 (2010).

67. Moiseev, S. A., Andrianov, S. N., and Gubaidullin, F. F. Efficient multimode quantum memory based on photon echo in an optimal qed cavity. Phys. Rev. A 82, 022311 (2010).

68. Afzelius, M. and Simon, C. Impedance-matched cavity quantum memory. Phys. Rev. A 82, 022310 (2010).

69. Sabooni, M., Li, Q., Kröll, S., S., and Rippe, L. Efficient quantum memory using a weakly absorbing sample. Phys. Rev. Lett. 110, 133604 (2013).

70. Jobez, P. et al. Cavity-enhanced storage in an optical spin-wave memory. New Journal of Physics 16, 083005- (2014).

71. Gündoğan, M., Ledingham, P. M., Almasi, A., Cristiani, M., and de Riedmatten, H. Quantum storage of a photonic polarization qubit in a solid. Phys. Rev. Lett. 108, 190504- (2012).

72. Sabooni, M. et al. Storage and recall of weak coherent optical pulses with an efficiency of 25 Phys. Rev. Lett. 105, 060501- (2010). 
73. Maring, N. et al. Storage of up-converted telecom photons in a doped crystal. New Journal of Physics 16, 113021-(2014).

74. Usmani, I., Afzelius, M., de Riedmatten, H., and Gisin, N. Mapping multiple photonic qubits into and out of one solid-state atomic ensemble. Nat Commun 1, 12- (2010).

75. Saglamyurek, E. et al. An integrated processor for photonic quantum states using a broadband lightmatter interface. New Journal of Physics 16, 065019- (2014).

76. Jin, J. et al. Two-photon interference of weak coherent laser pulses recalled from separate solid-state quantum memories. Nat Commun 4, - (2013).

77. Clausen, C., Bussières, F., Afzelius, M., and Gisin, N. Quantum storage of heralded polarization qubits in birefringent and anisotropically absorbing materials. Phys. Rev. Lett. 108, 190503-(2012).

78. Zhou, Z.-Q., Lin, W.-B., Yang, M., Li, C.-F., and Guo, G.-C. Realization of reliable solidstate quantum memory for photonic polarization qubit. Phys. Rev. Lett. 108, 190505- (2012).

79. Saglamyurek, E. et al. Conditional detection of pure quantum states of light after storage in a Tm-doped waveguide. Phys. Rev. Lett. 108, 083602- (2012).

80. Usmani, I. et al. Heralded quantum entanglement between two crystals. Nat Photon 6, 234-237 (2012).

81. Bussières, F. et al. Quantum teleportation from a telecom-wavelength photon to a solid-state quantum memory. Nat Photon 8, 775-778 (2014).

82. Zhou, Z.-Q. et al. Quantum storage of high-dimensional orbital-angular-momentum entanglement in a crystal. arXiv:1412.5243 (2014).

83. Longdell, J. J., Fraval, E., Sellars, M. J., and Manson, N. B. Stopped light with storage times greater than one second using electromagnetically induced transparency in a solid. Phys. Rev. Lett. 95, 063601-4 (2005).

84. Fekete, J., Rieländer, D., Cristiani, M., and de Riedmatten, H. Ultranarrow-band photonpair source compatible with solid state quantum memories and telecommunication networks. Phys. Rev. Lett. 110, 220502- (2013).

85. Saglamyurek, E. et al. Quantum storage of entangled telecom-wavelength photons in an erbium-doped optical fibre. Nature Photon. doi:10.1038/nphoton.2014.311 (2015).

86. Ledingham, P. M., Naylor, W. R., Longdell, J. J., Beavan, S. E., and Sellars, M. J. Nonclassical photon streams using rephased amplified spontaneous emission. Phys. Rev. A 81, 012301- (2010).

87. Sekatski, P., Sangouard, N., Gisin, N., de Riedmatten, H., and Afzelius, M. Photon-pair source with controllable delay based on shaped inhomogeneous broadening of rare-earthmetal-doped solids. Phys. Rev. A 83, 053840- (2011).

88. Beavan, S. E., Hedges, M. P., and Sellars, M. J. Demonstration of photon-echo rephasing of spontaneous emission. Phys. Rev. Lett. 109, 093603- (2012).

89. Ledingham, P. M., Naylor, W. R., and Longdell, J. J. Experimental realization of light with time-separated correlations by rephasing amplified spontaneous emission. Phys. Rev. Lett. 109, 093602- (2012).

90. Bonarota, M., Gouët, J.-L. L., and Chanelière, T. Highly multimode storage in a crystal. New Journal of Physics 13, 013013- (2011).

91. Ou, Z. Y. and Lu, Y. J. Cavity enhanced spontaneous parametric down-conversion for the prolongation of correlation time between conjugate photons. Phys. Rev. Lett. 83, 2556(1999).

92. Pomarico, E. et al. Waveguide-based OPO source of entangled photon pairs. New Journal of Physics 11, 113042 (2009).

93. Chuu, C.-S., Yin, G. Y., and Harris, S. E. A miniature ultrabright source of temporally long, narrowband biphotons. Appl. Phys. Lett. 101, 051108-4 (2012).

94. Förtsch, M. et al. A versatile source of single photons for quantum information processing. Nat Commun 4, 1818- (2013).

95. Kaiser, F. et al. A versatile source of polarization entangled photons for quantum network applications. Laser Phys. Lett. 10, 045202- (2013).

96. Chanelière, T. et al. Storage and retrieval of single photons transmitted between remote quantum memories. Nature 438, 833-836 (2005). 
97. Neergaard-Nielsen, J. S., Nielsen, B. M., Takahashi, H., Vistnes, A. I., and Polzik, E. S. High purity bright single photon source. Opt. Express 15, 7940-7949 (2007).

98. Bao, X.-H. et al. Generation of narrow-band polarization-entangled photon pairs for atomic quantum memories. Phys. Rev. Lett. 101, 190501-4 (2008).

99. Scholz, M., Koch, L., and Benson, O. Statistics of narrow-band single photons for quantum memories generated by ultrabright cavity-enhanced parametric down-conversion. Phys. Rev. Lett. 102, 063603-4 (2009).

100. Haase, A., Piro, N., Eschner, J., and Mitchell, M. W. Tunable narrowband entangled photon pair source for resonant single-photon single-atom interaction. Opt. Lett. 34, 55-57 (2009).

101. Wolfgramm, F., de Icaza Astiz, Y. A., Beduini, F. A., Cer, A., and Mitchell, M. W. Atomresonant heralded single photons by interaction-free measurement. Phys. Rev. Lett. 106, 053602- (2011).

102. Tanzilli, S. et al. Highly efficient photon-pair source using periodically poled lithium niobate waveguide. Electronics Letters 37, 26 (2001).

103. Halder, M. et al. High coherence photon pair source for quantum communication. New Journal of Physics 10, 023027- (2008).

104. Lettow, R. et al. Quantum interference of tunably indistinguishable photons from remote organic molecules. Phys. Rev. Lett. 104, 123605- (2010).

105. Tanzilli, S. et al. A photonic quantum information interface. Nature 437, 116-120 (2005).

106. Albrecht, B., Farrera, P., Fernandez-Gonzalvo, X., Cristiani, M., and de Riedmatten, H. A waveguide frequency converter connecting rubidium-based quantum memories to the telecom c-band. Nat Commun 5, - (2014).

107. Clausen, C. et al. A source of polarization-entangled photon pairs interfacing quantum memories with telecom photons. New Journal of Physics 16, 093058 (2014).

108. Christ, A., Laiho, K., Eckstein, A., Cassemiro, K. N., and Silberhorn, C. Probing multimode squeezing with correlation functions. New Journal of Physics 13, 033027- (2011).

109. Pomarico, E., Sanguinetti, B., Osorio, C. I., Herrmann, H., and Thew, R. T. Engineering integrated pure narrow-band photon sources. New Journal of Physics 14, 033008 (2012).

110. Franson, J. D. Bell inequality for position and time. Phys. Rev. Lett. 62, 2205-2208 (1989).

111. Clauser, J. F., Horne, M. A., Shimony, A., and Holt, R. A. Proposed experiment to test local hidden-variable theories. Phys. Rev. Lett. 23, 880-884 (1969).

112. van Enk, S. J. Single-particle entanglement. Phys. Rev. A 72, 064306- (2005).

113. Choi, K. S., Deng, H., Laurat, J., and Kimble, H. J. Mapping photonic entanglement into and out of a quantum memory. Nature 452, 67-71 (2008).

114. Chou, C. W. et al. Measurement-induced entanglement for excitation stored in remote atomic ensembles. Nature 438, 828-832 (2005).

115. Nilsson, M., Rippe, L., Kröll, S., Klieber, R., and Suter, D. Hole-burning techniques for isolation and study of individual hyperfine transitions in inhomogeneously broadened solids demonstrated in $\operatorname{Pr}^{3+}: \mathrm{Y}_{2} \mathrm{SiO}_{5}$. Phys. Rev. B 70, 214116-11 (2004).

116. McAuslan, D. L., Taylor, L. R., and Longdell, J. J. Using quantum memory techniques for optical detection of ultrasound. Applied Physics Letters 101, - (2012).

117. Afzelius, M. et al. Demonstration of atomic frequency comb memory for light with spinwave storage. Phys. Rev. Lett. 104, 040503- (2010).

118. Gündoğan, M., Mazzera, M., Ledingham, P. M., Cristiani, M., and de Riedmatten, H. Coherent storage of temporally multimode light using a spin-wave atomic frequency comb memory. New Journal of Physics 15, 045012- (2013).

119. Zhang, H. et al. Slow light for deep tissue imaging with ultrasound modulation. Applied Physics Letters 100, - (2012).

120. Beavan, S. E., Goldschmidt, E. A., and Sellars, M. J. Demonstration of a dynamic bandpass frequency filter in a rare-earth ion-doped crystal. J. Opt. Soc. Am. B 30, 1173-1177 (2013).

121. Timoney, N., Usmani, I., Jobez, P., Afzelius, M., and Gisin, N. Single-photon-level optical storage in a solid-state spin-wave memory. Phys. Rev. A 88, 022324- (2013).

122. Jobez, P, Laplane , C., Timoney, N., Gisin, N., Ferrier, A., Goldner, P. and Afzelius, M. Coherent spin control at the quantum level in an ensemble-based optical Memory arXiv:1501.03981(2015). 
123. Gündoğan, M. , Ledingham, P.M., Kutluer, K., Mazzera , M., and de Riedmatten, H. A solid-state quantum memory for time-bin qubits arXiv:1501.03980 (2015).

124. Johnsson, M. and Mølmer, K. Storing quantum information in a solid using dark-state polaritons. Phys. Rev. A 70, 032320 (2004).

125. Heshami, K., Sangouard, N., Mináŕ, J., de Riedmatten, H., and Simon, C. Precision requirements for spin-echo-based quantum memories. Phys. Rev. A 83, 032315 (2011).

126. Thiel, C., Sinclair, N., Tittel, W., and Cone, R. $\mathrm{Tm}^{3+}: \mathrm{Y}_{3} \mathrm{Ga}_{5} \mathrm{O}_{12}$ materials for spectrally multiplexed quantum memories. Phys. Rev. Lett. 113, 160501- (2014).

127. Zhong, T., Hartz, A., Miyazono, E., and Faraon, A. Towards coupling rare earth ions to a $\mathrm{Y}_{2} \mathrm{SiO}_{5}$ nanophotonic resonator. In: OSA Technical Digest (online), FTh1B.5- (Optical Society of America, San Jose, California, 2014).

128. Kolesov, R. et al. Optical detection of a single rare-earth ion in a crystal. Nat Commun 3, 1029- (2012).

129. Utikal, T. et al. Spectroscopic detection and state preparation of a single praseodymium ion in a crystal. Nat Commun 5, - (2014).

130. Staudt, M. U. et al. Coupling of an erbium spin ensemble to a superconducting resonator. Journal of Physics B: Atomic, Molecular and Optical Physics 45, 124019- (2012).

131. Probst, S. et al. Anisotropic rare-earth spin ensemble strongly coupled to a superconducting resonator. Phys. Rev. Lett. 110, 157001- (2013). 